\title{
MULTIPLICITY THEOREMS \\ FOR RESONANT AND SUPERLINEAR NONHOMOGENEOUS ELLIPTIC EQUATIONS
}

\author{
Nikolaos S. Papageorgiou - Vicenţiu D. Rădulescu
}

(Submitted by M. Clapp)

\begin{abstract}
We consider nonlinear elliptic equations driven by the sum of a $p$-Laplacian $(p>2)$ and a Laplacian. We consider two distinct cases. In the first one, the reaction $f(z, \cdot)$ is $(p-1)$-linear near $\pm \infty$ and resonant with respect to a nonprincipal variational eigenvalue of $\left(-\Delta_{p}, W_{0}^{1, p}(\Omega)\right)$. We prove a multiplicity theorem producing three nontrivial solutions. In the second case, the reaction $f(z, \cdot)$ is $(p-1)$-superlinear but does not satisfy the Ambrosetti-Rabinowitz condition. We prove two multiplicity theorems. In the first main result we produce six nontrivial solutions all with sign information and in the second theorem we have five nontrivial solutions. Our approach uses variational methods combined with the Morse theory, truncation methods, and comparison techniques.
\end{abstract}

\section{Introduction}

Let $\Omega \subseteq \mathbb{R}^{N}$ be a bounded domain with a $C^{2}$-boundary $\partial \Omega$. In this paper we study the following nonlinear nonhomogeneous Dirichlet problem:

(1.1) $\quad-\Delta_{p} u(z)-\Delta u(z)=f(z, u(z)) \quad$ in $\Omega, \quad u_{\left.\right|_{\partial \Omega}}=0, \quad 2<p<\infty$.

2010 Mathematics Subject Classification. Primary: 35J20; Secondary: 35J60, 35J92, $58 \mathrm{E} 05$.

Key words and phrases. Resonance; multiple solution; superlinear reaction; nodal solutions; critical groups.

The authors wish to thank a knowledgeable referee for his/her corrections and helpful remarks.

V.D. Rădulescu was supported by Grant CNCS-PCCA-23/2014. 
Here $\Delta_{p}$ denotes the $p$-Laplace differential operator defined by

$$
\Delta_{p} u=\operatorname{div}\left(|D u|^{p-2} D u\right) \quad \text { for all } u \in W_{0}^{1, p}(\Omega) .
$$

If $p=2$, then $\Delta_{2}=\Delta=$ the Laplace differential operator.

The reaction $f$ is a Carathéodory function (that is, for all $x \in \mathbb{R}$, the mapping $z \mapsto f(z, x)$ is measurable and for almost all $z \in \Omega, x \mapsto f(z, x)$ is continuous). Additional regularity conditions on $f(z, \cdot)$ are introduced in order to produce extra solutions. We consider two distinct cases. In the first (Section 3), we assume that $f(z, \cdot)$ is $(p-1)$-linear near $\pm \infty$ and interacts with a nonprincipal variational eigenvalue of $\left(-\Delta_{p}, W_{0}^{1, p}(\Omega)\right)$ (resonant problem). We prove a multiplicity theorem, producing three nontrivial solutions two of which have constant sign. In the second case (Section 4), we deal with reaction $f(z, \cdot)$ which is $(p-1)$-superlinear near $\pm \infty$ but without satisfying the usual in such cases Ambrosetti-Rabinowitz condition (AR-condition for short). We also assume that $f(z, \cdot)$ has $z$-dependent zeros of constant sign. We prove a multiplicity theorem producing six nontrivial solutions, four of constant sign and two nodal (sign changing).

Recently nonhomogeneous nonlinear equations driven by the sum of a $p$ Laplacian and a Laplacian (a $(p, 2)$-equation for short), were studied by Cingolani and Degiovanni [7], Papageorgiou and Rădulescu [21], Papageorgiou and Smyrlis [22] and Sun [24]. All these works deal with equations that have a $(p-1)$ linear reaction and either they do not allow resonance (see [7]) or the resonance is with respect to the principal eigenvalue of $\left(-\Delta_{p}, W_{0}^{1, p}(\Omega)\right)$ (see [21], [22], [24]). Recall that for $p \neq 2$, we do not have a complete knowledge of the spectrum of $\left(-\Delta_{p}, W_{0}^{1, p}(\Omega)\right)$, the eigenspaces are not linear subspaces and we do not have a direct sum decomposition of $W_{0}^{1, p}(\Omega)$ in terms of them. All these facts, make problems resonant at higher parts of the spectrum difficult to deal with. On the other hand, problems with reactions which have zeros of constant sign, were investigated only in the context of $p$-Laplacian equations, by Bartsch, Liu and Weth [5] (constant zeros) and by Iturriaga, Massa, Sanchez and Ubilla [17] (variable zeros for a class of parametric equations). None of the aforementioned works produces six nontrivial solutions all with sign information.

Our approach is a combination of variational methods based on the critical point theory together with the Morse theory (critical groups) and truncation and comparison techniques. In the next section, for the convenience of the reader, we recall the main mathematical tools which we will use in the sequel.

\section{Mathematical background}

Let $X$ be a Banach space and $X^{*}$ its topological dual. By $\langle\cdot, \cdot\rangle$ we denote the duality brackets for the pair $\left(X, X^{*}\right)$. Let $\varphi \in C^{1}(X)$. We say that $\varphi$ satisfies the Cerami condition (the C-condition for short), if the following holds: 
"Every sequence $\left\{u_{n}\right\}_{n \geq 1} \subseteq X$ such that $\left\{\varphi\left(u_{n}\right)\right\}_{n \geq 1} \subseteq \mathbb{R}$ is bounded and

$$
\left(1+\left\|u_{n}\right\|\right) \varphi^{\prime}\left(u_{n}\right) \rightarrow 0 \quad \text { in } X^{*},
$$

admits a strongly convergent subsequence".

This is a compactness type condition on the functional $\varphi$ which compensates for the fact that the underlying space $X$ is not locally compact (being in general infinite dimensional). The C-condition is more general than the more familiar Palais-Smale condition (PS-condition for short) and the two are equivalent if $\varphi$ is bounded below. The C-condition is a basic tool in proving a deformation theorem from which we can derive the minimax theory of certain critical values of $\varphi$. Prominent in that theory is the so-called "mountain pass theorem" due to Ambrosetti and Rabinowitz [3]. Here the theorem is presented in a slightly more general form with the C-condition replacing the PS-condition (see, for example, Gasinski and Papageorgiou [15, p. 648]).

Theorem 2.1. Assume that $\varphi \in C^{1}(X)$ satisfies the $C$-condition, $u_{0}, u_{1} \in X$, $\left\|u_{1}-u_{0}\right\|>\rho>0$

$$
\max \left\{\varphi\left(u_{0}\right), \varphi\left(u_{1}\right)\right\}<\inf \left[\varphi(u):\left\|u-u_{0}\right\|=\rho\right]=m_{\rho}
$$

and $c=\inf _{\gamma \in \Gamma} \max _{0 \leq t \leq 1} \varphi(\gamma(t))$ with $\Gamma=\left\{\gamma \in C([0,1], X): \gamma(0)=u_{0}, \gamma(1)=u_{1}\right\}$.

Then $c \geq m_{\rho}$ and $c$ is a critical value of $\varphi$.

In what follows by $|\cdot|$ we denote the norm of $\mathbb{R}^{N}$ and by $\|\cdot\|$ the norm of the Sobolev space $W_{0}^{1, p}(\Omega)$. By virtue of the Poincaré inequality, we have

$$
\|u\|=\|D u\|_{p} \quad \text { for all } u \in W_{0}^{1, p}(\Omega) .
$$

Let $1<p<\infty$ and let $A_{p}: W_{0}^{1, p}(\Omega) \rightarrow W^{-1, p^{\prime}}(\Omega)=W_{0}^{1, p}(\Omega)^{*}, 1 / p+1 / p^{\prime}=$ 1 , be the nonlinear map defined by

$$
\left\langle A_{p}(u), y\right\rangle=\int_{\Omega}|D u|^{p-2}(D u, D y)_{\mathbb{R}^{N}} d z \quad \text { for all } u, y \in W_{0}^{1, p}(\Omega) .
$$

When $p=2$, then we write $A_{2}=A$ and this map is linear. The next proposition summarizes the main properties of this map (see, for example, Papageorgiou and Kyritsi [20, p. 314]).

Proposition 2.2. The map $A_{p}: W_{0}^{1, p}(\Omega) \rightarrow W^{-1, p^{\prime}}(\Omega)$ is bounded (that is, maps bounded sets to bounded sets), demicontinuous, strictly monotone (hence maximal monotone too) and of type $(\mathrm{S})_{+}$, that is, if $u_{n} \stackrel{\mathrm{w}}{\longrightarrow} u$ in $W_{0}^{1, p}(\Omega)$ and

$$
\limsup _{n \rightarrow \infty}\left\langle A_{p}\left(u_{n}\right), u_{n}-u\right\rangle \leq 0
$$

then $u_{n} \rightarrow u$ in $W_{0}^{1, p}(\Omega)$. 
Also, we can view $A_{p}$ defined on the bigger Sobolev space $W^{1, p}(\Omega)$, that is $A_{p}: W^{1, p}(\Omega) \rightarrow W^{1, p}(\Omega)^{*}$. With a slight abuse of notation, we still denote the duality brackets by $\langle\cdot, \cdot\rangle$ and we have

$$
\langle A(u), y\rangle=\int_{\Omega}|D u|^{p-2}(D u, D y)_{\mathbb{R}^{N}} d z \quad \text { for all } u, y \in W^{1, p}(\Omega) .
$$

This map too is bounded, demicontinuous, maximal monotone and of type $(\mathrm{S})_{+}$.

In addition to the Sobolev space $W_{0}^{1, p}(\Omega)$, we will also use the Banach space $C_{0}^{1}(\bar{\Omega})=\left\{u \in C^{1}(\bar{\Omega}): u_{\left.\right|_{\partial \Omega}}=0\right\}$. This is an ordered Banach space with positive cone $C_{+}=\left\{u \in C_{0}^{1}(\bar{\Omega}): u(z) \geq 0\right.$ for all $\left.z \in \bar{\Omega}\right\}$. This cone has a nonempty interior, given by

$$
\operatorname{int} C_{+}=\left\{u \in C_{+}: u(z)>0 \text { for all } z \in \Omega, \frac{\partial u}{\partial n}(z)<0 \text { for all } z \in \partial \Omega\right\},
$$

where $n(\cdot)$ denotes the outward unit normal on $\partial \Omega$.

By $p^{*}$ we denote the critical Sobolev exponent. So,

$$
p^{*}= \begin{cases}\frac{N p}{N-p} & \text { if } p<N \\ +\infty & \text { if } N \leq p\end{cases}
$$

Let $f_{0}: \Omega \times \mathbb{R} \rightarrow \mathbb{R}$ be a Carathéodory function with subcritical growth in the $x$-variable, that is,

$$
\left|f_{0}(z, x)\right| \leq a_{0}(z)\left(1+|x|^{r-1}\right) \quad \text { for a.a. } z \in \Omega \text {, all } x \in \mathbb{R},
$$

with $a_{0} \in L^{\infty}(\Omega)_{+}, 1<r<p^{*}$. Let $F_{0}(z, x)=\int_{0}^{x} f_{0}(z, s) d s$ and $\varphi_{0}: W_{0}^{1, p}(\Omega) \rightarrow \mathbb{R}$ $(2<p<\infty)$ be the $C^{1}$-functional defined by

$$
\varphi_{0}(u)=\frac{1}{p}\|D u\|_{p}^{p}+\frac{1}{2}\|D u\|_{2}^{2}-\int_{\Omega} F_{0}(z, u(z)) d z \quad \text { for all } u \in W_{0}^{1, p}(\Omega) .
$$

The following proposition is a special case of a more general result of Gasinski and Papageorgiou [16] and essentially is a consequence of the regularity results of Lieberman [19].

Proposition 2.3. Assume that $u_{0} \in W_{0}^{1, p}(\Omega)$ is a local $C_{0}^{1}(\bar{\Omega})$-minimizer of $\varphi_{0}$, that is, there exists $\rho_{0}>0$ such that

$$
\varphi_{0}\left(u_{0}\right) \leq \varphi_{0}\left(u_{0}+h\right) \quad \text { for all } h \in C_{0}^{1}(\bar{\Omega}),\|h\|_{C_{0}^{1}(\bar{\Omega})} \leq \rho_{0} .
$$

Then $u_{0} \in C_{0}^{1, \alpha}(\bar{\Omega})$ for some $\alpha \in(0,1)$ and $u_{0}$ is also a local $W_{0}^{1, p}(\Omega)$-minimizer of $\varphi_{0}$, that is, there exists $\rho_{1}>0$ such that

$$
\varphi_{0}\left(u_{0}\right) \leq \varphi_{0}\left(u_{0}+h\right) \quad \text { for all } h \in W_{0}^{1, p}(\Omega),\|h\| \leq \rho_{1} .
$$

Let $\eta, \widehat{\eta} \in L^{\infty}(\Omega)$. We say $\eta \prec \widehat{\eta}$ if and only if for every compact $K \subseteq \Omega$, we can find $\varepsilon=\varepsilon_{K}>0$ such that

$$
\eta(z)+\varepsilon \leq \widehat{\eta}(z) \quad \text { for a.a. } z \in K .
$$


Clearly, if $\eta, \widehat{\eta} \in C(\Omega)$ and $\eta(z)<\widehat{\eta}(z)$ for all $z \in \Omega$, then $\eta \prec \widehat{\eta}$.

A simple modification of the proof of Proposition 2.6 of Arcoya and Ruiz [4], in order to accommodate the presence of the extra linear term $-\Delta u$, leads to the following strong comparison property.

Proposition 2.4. Assume that $\beta \geq 0, \eta, \widehat{\eta} \in L^{\infty}(\Omega), \eta \prec \widehat{\eta}$ and $u \in C_{0}^{1}(\bar{\Omega})$, $v \in \operatorname{int} C_{+}$satisfy

$$
\begin{aligned}
& -\Delta_{p} u(z)-\Delta u(z)+\beta|u(z)|^{p-2} u(z)=\eta(z) \text { in } \Omega, \\
& -\Delta_{p} v(z)-\Delta v(z)+\beta v(z)^{p-1}=\widehat{\eta}(z) \text { in } \Omega .
\end{aligned}
$$

Then $v-u \in \operatorname{int} C_{+}$.

As we have already mentioned in Introduction, our approach will use also the Morse theory (critical groups). So, next we recall some basic definitions and facts from Morse theory. Given a topological pair $\left(Y_{1}, Y_{2}\right)$ with $Y_{2} \subseteq Y_{1} \subseteq X$, for every integer $k \geq 0$ by $H_{k}\left(Y_{1}, Y_{2}\right)$ we denote the $k^{\text {th }}$-relative singular homology group with integer coefficients for the pair $\left(Y_{1}, Y_{2}\right)$. Given $\varphi \in C^{1}(X)$ and $c \in \mathbb{R}$, we introduce the following sets:

$$
\begin{aligned}
\varphi^{c} & =\{u \in X: \varphi(u) \leq c\}, \\
K_{\varphi} & =\left\{u \in X: \varphi^{\prime}(u)=0\right\}, \\
K_{\varphi}^{c} & =\left\{u \in K_{\varphi}: \varphi(u)=c\right\} .
\end{aligned}
$$

If $u \in K_{\varphi}^{c}$ is isolated, then the critical groups of $\varphi$ at $u$ are defined by

$$
C_{k}(\varphi, u)=H_{k}\left(\varphi^{c} \cap U, \varphi^{c} \cap U \backslash\{u\}\right) \quad \text { for all } k \geq 0,
$$

where $U$ is a neighbourhood of $u$ such that $K_{\varphi} \cap \varphi^{c} \cap U=\{u\}$. The excision property of singular homology implies that the above definition of critical groups is independent of the choice of the neighbourhood $U$.

Suppose that $\varphi \in C^{1}(X)$ satisfies the C-condition and inf $\varphi\left(K_{\varphi}\right)>-\infty$. Let $c<\inf \varphi\left(K_{\varphi}\right)$. The critical groups of $\varphi$ at infinity are defined by

$$
C_{k}(\varphi, \infty)=H_{k}\left(X, \varphi^{c}\right) \text { for all } k \geq 0 .
$$

The second deformation theorem (see, for example, Gasinski and Papageorgiou $[15$, p. 628]) implies that the above definition of critical groups at infinity is independent of the choice of the level $c<\inf \varphi\left(K_{\varphi}\right)$.

Suppose that $K_{\varphi}$ is finite. We introduce

$$
\begin{aligned}
& M(t, u)=\sum_{k \geq 0} \operatorname{rank} C_{k}(\varphi, u) t^{k} \quad \text { for all } t \in \mathbb{R}, \text { all } u \in K_{\varphi}, \\
& P(t, \infty)=\sum_{k \geq 0} \operatorname{rank} C_{k}(\varphi, \infty) t^{k} \quad \text { for all } t \in \mathbb{R} .
\end{aligned}
$$


The Morse relation says that

$$
\sum_{u \in K_{\varphi}} M(t, u)=P(t, \infty)+(1+t) Q(t),
$$

where $Q(t)=\sum_{k>0} \beta_{k} t^{k}$ is a formal series in $t \in \mathbb{R}$ with nonnegative integer coefficients.

Next, let us recall some basic facts concerning the spectrum of $\left(-\Delta_{p}, W_{0}^{1, p}(\Omega)\right)$. So, we consider the following nonlinear eigenvalue problem:

$$
-\Delta_{p} u(z)=\widehat{\lambda}|u(z)|^{p-2} u(z) \quad \text { in } \Omega, u_{\left.\right|_{\partial \Omega}}=0 .
$$

A number $\widehat{\lambda} \in \mathbb{R}$ is an eigenvalue of $\left(-\Delta_{p}, W_{0}^{1, p}(\Omega)\right)$, if problem (2.2) admits a nontrivial solution $\widehat{u}$. The nontrivial solution $\widehat{u}$ is an eigenfunction corresponding to the eigenvalue $\widehat{\lambda}$. The smallest eigenvalue is denoted by $\widehat{\lambda}_{1}(p)$ and has the following properties:

- $\hat{\lambda}_{1}(p)>0$ and it is isolated;

- $\hat{\lambda}_{1}(p)$ is simple (that is, if $\widehat{u}, \widehat{v}$ are eigenfunctions corresponding to the eigenvalue $\widehat{\lambda}_{1}(p)$, then $\widehat{u}=\xi \widehat{v}$ for some $\xi \neq 0$ );

- we have

$$
\widehat{\lambda}_{1}(p)=\inf \left[\frac{\|D u\|_{p}^{p}}{\|u\|_{p}^{p}}: u \in W_{0}^{1, p}(\Omega), u \neq 0\right] .
$$

In (2.3) the infimum is realized on the one-dimensional eigenspace corresponding to $\widehat{\lambda}_{1}(p)>0$. In what follows, by $\widehat{u}_{1}(p)$ we denote the $L^{p}$-normalized (that is, $\left.\left\|\widehat{u}_{1}(p)\right\|_{p}=1\right)$ positive eigenfunction corresponding to $\widehat{\lambda}_{1}(p)$. The nonlinear regularity theory and the nonlinear maximum principle (see, for example, Gasinski and Papageorgiou [15, pp. 737-738]), imply that $\widehat{u}_{1}(p) \in \operatorname{int} C_{+}$. Since the spectrum $\sigma(p)$ of $\left(-\Delta_{p}, W_{0}^{1, p}(\Omega)\right)$ is closed and $\widehat{\lambda}_{1}(p)>0$ is isolated, then the second eigenvalue is well-defined by

$$
\widehat{\lambda}_{2}(p)=\inf \left[\widehat{\lambda} \in \sigma(p): \widehat{\lambda}>\widehat{\lambda}_{1}(p)\right] .
$$

Let ind $(A)$ be the $\mathbb{Z}_{2}$-cohomological index introduced by Fadell and Rabinowitz [13]. Employing the Lusternik-Schnirelmann minimax scheme, we can define a whole divergent sequence $\left\{\widehat{\lambda}_{k}(p)\right\}_{k \geq 1}$ of distinct eigenvalues of $\left(-\Delta_{p}, W_{0}^{1, p}(\Omega)\right)$ by setting

$$
\widehat{\lambda}_{k}(p)=\inf \left[\sup _{u \in A}\|D u\|_{p}^{p}: A \subseteq M \text { symmetric, } \operatorname{ind}(A) \geq k\right], \quad k \geq 1,
$$

where $M=\left\{u \in W_{0}^{1, p}(\Omega):\|u\|_{p}=1\right\}$ is a $C^{1}$-Banach manifold. For $k=1,2$, these eigenvalues are as above. We do not know if the sequence $\left\{\widehat{\lambda}_{k}(p)\right\}_{k \geq 1}$ exhausts $\sigma(p)$. This is the case if $N=1$ (ordinary differential equation) or if 
$p=2$ and $N \geq 2$ (linear eigenvalue problem). In the latter case, we have

$$
H_{0}^{1}(\Omega)=\overline{\bigoplus_{k \geq 1} E\left(\widehat{\lambda}_{k}(2)\right)}
$$

where $E\left(\widehat{\lambda}_{k}(2)\right)$ denotes the eigenspace corresponding to the eigenvalue $\widehat{\lambda}_{k}(2)$. We know that for every $k \geq 1, E\left(\widehat{\lambda}_{k}(2)\right)$ is finite dimensional, $E\left(\widehat{\lambda}_{k}(2)\right) \subseteq C_{0}^{1}(\bar{\Omega})$ and it has the "Unique Continuation Property" (UCP for short), that is, if $u \in E\left(\widehat{\lambda}_{k}(2)\right)$ and vanishes on a set of positive measure, then $u \equiv 0$.

We mention that if $\widehat{u}$ is an eigenfunction corresponding to an eigenvalue $\widehat{\lambda}_{m}(p)$ with $m \geq 2$, then $\widehat{u} \in C_{0}^{1}(\bar{\Omega})$ (nonlinear regularity theory) and $\widehat{u}$ is nodal (that is, sign changing). In fact, if $p=2,\left\{\widehat{u}_{n}\right\}_{n \geq 1} \subseteq H_{0}^{1}(\Omega)$ are the eigenfunctions corresponding to the eigenvalues $\left\{\widehat{\lambda}_{k}(2)\right\}_{k \geq 1}$ and $S_{n}=\{z \in \Omega$ : $\left.\widehat{u}_{n}(z)=0\right\}$, then $\Omega \backslash S_{n}$ has at most $n$-components (the Courant nodal domain theorem, see, for example, Gasinski and Papageorgiou [15, p. 797]).

For $x \in \mathbb{R}$, let $x^{ \pm}=\max \{ \pm x, 0\}$. Then for $u \in W_{0}^{1, p}(\Omega)$, we set $u^{ \pm}(\cdot)=$ $u(\cdot)^{ \pm}$. We know that

$$
u^{ \pm} \in W_{0}^{1, p}(\Omega), \quad u=u^{+}-u^{-}, \quad|u|=u^{+}+u^{-} .
$$

By $|\cdot|_{N}$ we denote the Lebesgue measure on $\mathbb{R}^{N}$. Finally, if $h: \Omega \times \mathbb{R} \rightarrow \mathbb{R}$ is a measurable function (for example, a Carathéodory function), then we set

$$
N_{h}(u)(\cdot)=h(\cdot, u(\cdot)) \quad \text { for all } u \in W_{0}^{1, p}(\Omega),
$$

the Nemytskil (superposition) operator corresponding to the function $h$. Evidently, $z \mapsto N_{h}(u)(z)=h(z, u(z))$ is measurable.

\section{Resonant problems}

In this section we study problem (1.1) when the reaction $f(z, \cdot)$ is $(p-1)$ linear near $\pm \infty$ and resonant with respect to a nonprincipal eigenvalue of $\left(-\Delta_{p}\right.$, $\left.W_{0}^{1, p}(\Omega)\right)$. So, we impose the following conditions on the function $f$ :

$\left(\mathrm{H}_{1}\right) f: \Omega \times \mathbb{R} \rightarrow \mathbb{R}$ is a Carathéodory function such that $f(z, 0)=0$ for almost all $z \in \Omega$ and

(i) $|f(z, x)| \leq a(z)\left(1+|x|^{p-1}\right)$ for almost all $z \in \Omega$, all $x \in \mathbb{R}$, with $a \in L^{\infty}(\Omega)_{+}$

(ii) $\lim _{x \rightarrow \pm \infty} \frac{f(z, x)}{|x|^{p-2} x}=\widehat{\lambda}_{m}(p)$ uniformly for almost all $z \in \Omega$ and with $m \geq 2$;

(iii) if $F(z, x)=\int_{0}^{x} f(z, s) d s$, then there exists $\tau \in(2, p)$ such that $0<\eta_{0} \leq \liminf _{x \rightarrow \pm \infty} \frac{p F(z, x)-f(z, x) x}{|x|^{\tau}}$ uniformly for almost all $z \in \Omega ;$ 
(iv) there exists $\vartheta \in L^{\infty}(\Omega)+$ such that

$$
\begin{array}{rlrl}
\vartheta(z) & \leq \widehat{\lambda}_{1}(2) & \text { a.e. in } \Omega, \vartheta \not \equiv \widehat{\lambda}_{1}(2), \\
\limsup _{x \rightarrow 0} \frac{2 F(z, x)}{x^{2}} & \leq \vartheta(z) \quad \text { uniformly for a.a. } z \in \Omega
\end{array}
$$

(v) for every $\rho>0$, there exists $\xi_{\rho}>0$ such that $f(z, x) x+\xi_{\rho}|x|^{p} \geq 0$ for almost all $z \in \Omega$, all $|x| \leq \rho$.

REMARK 3.1. If there exists $\delta>0$ such that $f(z, x) x \geq 0$ for almost all $z \in \Omega$, all $|x| \leq \delta$, then hypothesis $\left(\mathrm{H}_{1}\right)(\mathrm{v})$ is automatically satisfied.

EXAMPLE 3.2. The following function satisfies hypotheses $\left(\mathrm{H}_{1}\right)$. For the sake of simplicity we drop the $z$-dependence:

$$
f(x)= \begin{cases}\vartheta x+\widehat{\lambda}_{m}(p)|x|^{q-2} x & \text { if }|x| \leq 1 \\ \widehat{\lambda}_{m}(p)|x|^{p-2} x+\vartheta|x|^{\tau-2} x & \text { if }|x|>1\end{cases}
$$

with $\tau, q>2, \tau<p$ and $\vartheta \in\left(0, \widehat{\lambda}_{1}(2)\right)$.

We set $f_{ \pm}(z, x)=f\left(z, \pm x^{ \pm}\right)$. These are Carathéodory functions. Let

$$
F_{ \pm}(z, x)=\int_{0}^{x} f_{ \pm}(z, s) d s
$$

and let $\varphi_{ \pm}: W_{0}^{1, p}(\Omega) \rightarrow \mathbb{R}$ be the $C^{1}$-functionals defined by

$$
\varphi_{ \pm}(u)=\frac{1}{p}\|D u\|_{p}^{p}+\frac{1}{2}\|D u\|_{2}^{2}-\int_{\Omega} F_{ \pm}(z, u(z)) d z \quad \text { for all } u \in W_{0}^{1, p}(\Omega) .
$$

Also, let $\varphi: W_{0}^{1, p}(\Omega) \rightarrow \mathbb{R}$ be the energy functional for problem (1.1) defined by

$$
\varphi(u)=\frac{1}{p}\|D u\|_{p}^{p}+\frac{1}{2}\|D u\|_{2}^{2}-\int_{\Omega} F(z, u(z)) d z \quad \text { for all } u \in W_{0}^{1, p}(\Omega) .
$$

Evidently $\varphi \in C^{1}\left(W_{0}^{1, p}(\Omega)\right)$.

Proposition 3.3. Assume that hypotheses $\left(\mathrm{H}_{1}\right)$ (i), (ii) hold. Then the functionals $\varphi_{ \pm}$satisfy the $C$-condition.

Proof. We do the proof for $\varphi_{+}$, the proof for $\varphi_{-}$being similar. So, let $\left\{u_{n}\right\}_{n \geq 1} \subseteq W_{0}^{1, p}(\Omega)$ be such that

$$
\begin{array}{ll}
\left|\varphi_{+}\left(u_{n}\right)\right| \leq M_{1} & \text { for some } M_{1}>0, \text { all } n \geq 1, \\
\left(1+\left\|u_{n}\right\|\right) \varphi^{\prime}\left(u_{n}\right) \rightarrow 0 & \text { in } W^{-1, p^{\prime}}(\Omega) \text { as } n \rightarrow \infty .
\end{array}
$$

From (3.2) we have

$$
\left|\left\langle A_{p}\left(u_{n}\right), h\right\rangle+\left\langle A\left(u_{n}\right), h\right\rangle-\int_{\Omega} f_{+}\left(z, u_{n}\right) h d z\right| \leq \frac{\varepsilon_{n}\|h\|}{1+\left\|u_{n}\right\|}
$$

for all $h \in W_{0}^{1, p}(\Omega)$ with $\varepsilon_{n} \rightarrow 0^{+}$. 
In (3.3) we choose $h=-u_{n}^{-} \in W_{0}^{1, p}(\Omega)$. Then

(3.4) $\left\|D u_{n}^{-}\right\|_{p}^{p}+\left\|D u_{n}^{-}\right\|_{2}^{2} \leq \varepsilon_{n}$ for all $n \geq 1$

$$
\Rightarrow u_{n}^{-} \rightarrow 0 \text { in } W_{0}^{1, p}(\Omega) \text { as } n \rightarrow \infty .
$$

Claim. $\left\{u_{n}^{+}\right\}_{n \geq 1} \subseteq W_{0}^{1, p}(\Omega)$ is bounded.

We argue indirectly. So, suppose that $\left\{u_{n}^{+}\right\}_{n \geq 1} \subseteq W_{0}^{1, p}(\Omega)$ is not bounded. We may assume that $\left\|u_{n}^{+}\right\| \rightarrow \infty$. Let $y_{n}=u_{n}^{+} /\left\|u_{n}^{+}\right\|, n \geq 1$. Then $\left\|y_{n}\right\|=1$, $y_{n} \geq 0$ for all $n \geq 1$. So, by passing to a subsequence if necessary, we may assume that

$$
y_{n} \stackrel{\mathrm{w}}{\longrightarrow} y \quad \text { in } W_{0}^{1, p}(\Omega) \text { and } \quad y_{n} \rightarrow y \quad \text { in } L^{p}(\Omega) \text {, with } y \geq 0 .
$$

From (3.3) and (3.4), we have

$$
\left|\left\langle A_{p}\left(y_{n}\right), h\right\rangle+\frac{1}{\left\|u_{n}^{+}\right\|^{p-2}}\left\langle A\left(y_{n}\right), h\right\rangle-\int_{\Omega} \frac{f_{+}\left(z, u_{n}\right)}{\left\|u_{n}^{+}\right\|^{p-1}} h d z\right| \leq \varepsilon_{n}^{\prime}\|h\|
$$

with $\varepsilon_{n}^{\prime} \rightarrow 0^{+}$as $n \rightarrow \infty$.

In (3.6) we choose $h=y_{n}-y \in W_{0}^{1, p}(\Omega)$, pass to the limit as $n \rightarrow \infty$ and use (3.5). Then

$$
\begin{aligned}
& \lim _{n \rightarrow \infty}\left\langle A_{p}\left(y_{n}\right), y_{n}-y\right\rangle=0 \quad(\text { recall } p>2), \\
& \Rightarrow y_{n} \rightarrow y \text { in } W_{0}^{1, p}(\Omega) \quad(\text { see Proposition } 2.2), \text { hence }\|y\|=1, y \geq 0 .
\end{aligned}
$$

Hypothesis $\left(\mathrm{H}_{1}\right)(\mathrm{i})$ implies that

$$
\left\{\frac{N_{f_{+}}\left(u_{n}\right)}{\left\|u_{n}^{+}\right\|^{p-1}}=\frac{N_{f}\left(u_{n}^{+}\right)}{\left\|u_{n}^{+}\right\|^{p-1}}\right\}_{n \geq 1} \subseteq L^{p^{\prime}}(\Omega) \quad \text { is bounded. }
$$

So, we may assume that

$$
\frac{N_{f_{+}}\left(u_{n}\right)}{\left\|u_{n}^{+}\right\|^{p-1}} \stackrel{\mathrm{w}}{\longrightarrow} g \text { in } L^{p^{\prime}}(\Omega) \text { as } n \rightarrow \infty .
$$

By virtue of hypothesis $\left(\mathrm{H}_{1}\right)(\mathrm{ii})$, we have

$$
g=\widehat{\lambda}_{m}(p) y^{p-1}
$$

(see Aizicovici, Papageorgiou and Staicu [1], proof of Proposition 30). Then, if in (3.6) we pass to the limit as $n \rightarrow \infty$ and use (3.7)-(3.9) and the fact that $p>2$, we obtain

$$
\begin{aligned}
\left\langle A_{p}(y), h\right\rangle=\widehat{\lambda}_{m}(p) \int_{\Omega} y^{p-1} h d z \text { for all } h \in & W_{0}^{1, p}(\Omega), \\
& \Rightarrow A_{p}(y)=\widehat{\lambda}_{m}(p) y^{p-1} .
\end{aligned}
$$

Since $m \geq 2$, from (3.10) we infer that $y \equiv 0$ or $y$ is nodal, both cases contradict (3.7). This proves Claim. 
From (3.4) and Claim, it follows that $\left\{u_{n}\right\}_{n \geq 1} \subseteq W_{0}^{1, p}(\Omega)$ is bounded and so we may assume that

$$
u_{n} \stackrel{\mathrm{w}}{\longrightarrow} u \quad \text { in } W_{0}^{1, p}(\Omega) \quad \text { and } \quad u_{n} \rightarrow u \quad \text { in } L^{p}(\Omega) .
$$

In (3.3) we choose $h=u_{n}-u \in W_{0}^{1, p}(\Omega)$, pass to the limit as $n \rightarrow \infty$ and use (3.11). Then

$$
\begin{aligned}
& \lim _{n \rightarrow \infty}\left[\left\langle A_{p}\left(u_{n}\right), u_{n}-u\right\rangle+\left\langle A\left(u_{n}\right), u_{n}-u\right\rangle\right]=0, \\
& \quad \Rightarrow \limsup _{n \rightarrow \infty}\left[\left\langle A_{p}\left(u_{n}\right), u_{n}-u\right\rangle+\left\langle A(u), u_{n}-u\right\rangle\right] \leq 0 \quad \text { (since A is monotone), } \\
& \quad \Rightarrow \limsup _{n \rightarrow \infty}\left\langle A_{p}\left(u_{n}\right), u_{n}-u\right\rangle \leq 0, \\
& \quad \Rightarrow u_{n} \rightarrow u \text { in } W_{0}^{1, p}(\Omega)
\end{aligned}
$$

This proves that the functional $\varphi_{+}$satisfies the C-condition. Similarly for the functional $\varphi_{-}$.

Proposition 3.4. If hypotheses $\left(\mathrm{H}_{1}\right)$ (i)-(iii) hold, then the functional $\varphi$ satisfies the $C$-condition.

Proof. Let $\left\{u_{n}\right\}_{n \geq 1} \subseteq W_{0}^{1, p}(\Omega)$ be a sequence such that

$$
\begin{array}{ll}
\left|\varphi\left(u_{n}\right)\right| \leq M_{2} & \text { for some } M_{2}>0, \text { all } n \geq 1, \\
\left(1+\left\|u_{n}\right\|\right) \varphi^{\prime}\left(u_{n}\right) \rightarrow 0 & \text { in } W^{-1, p^{\prime}}(\Omega) \text { as } n \rightarrow \infty
\end{array}
$$

From (3.13) we have

$$
\left|\left\langle A_{p}\left(u_{n}\right), h\right\rangle+\left\langle A\left(u_{n}\right), h\right\rangle-\int_{\Omega} f\left(z, u_{n}\right) h d z\right| \leq \frac{\varepsilon_{n}\|h\|}{1+\left\|u_{n}\right\|}
$$

for all $h \in W_{0}^{1, p}(\Omega)$ with $\varepsilon_{n} \rightarrow 0^{+}$. In (3.14) we choose $h=u_{n} \in W_{0}^{1, p}(\Omega)$ and obtain

$$
\left\|D u_{n}\right\|_{p}^{p}+\|D u\|_{2}^{2}-\int_{\Omega} f\left(z, u_{n}\right) u_{n} d z \leq \varepsilon_{n} \quad \text { for all } n \geq 1 .
$$

Also, from (3.12) we have

$$
-\left\|D u_{n}\right\|_{p}^{p}-\frac{p}{2}\left\|D u_{n}\right\|_{2}^{2}+\int_{\Omega} p F\left(z, u_{n}\right) d z \leq p M_{2} \quad \text { for all } n \geq 1 .
$$

Adding (3.15) and (3.16), we obtain

$$
\int_{\Omega}\left[p F\left(z, u_{n}\right)-f\left(z, u_{n}\right) u_{n}\right] d z \leq M_{3}+\left(\frac{p}{2}-1\right)\left\|D u_{n}\right\|_{2}^{2}
$$

for some $M_{3}>0$, all $n \geq 1$. 
By virtue of hypotheses $\left(\mathrm{H}_{1}\right)(\mathrm{i})$,(iii), we can find $\eta_{1} \in\left(0, \eta_{0}\right)$ and $c_{1}>0$ such that

$$
\eta_{1}|x|^{\tau}-c_{1} \leq p F(z, x)=f(z, x) x \quad \text { for a.a. } z \in \Omega \text {, all } x \in \mathbb{R},
$$

$$
\Rightarrow \eta_{1}\left\|u_{n}\right\|_{\tau}^{\tau} \leq M_{4}+\left(\frac{p}{2}-1\right)\left\|D u_{n}\right\|_{2}^{2} \quad \text { for some } M_{4}>0, \text { all } n \geq 1
$$

(see (3.17)).

Claim $\left\{u_{n}\right\}_{n \geq 1} \subseteq W_{0}^{1, p}(\Omega)$ is bounded.

Arguing by contradiction, suppose that Claim is not true. Then by passing to a subsequence if necessary, we may assume that $\left\|u_{n}\right\| \rightarrow \infty$. Let $y_{n}=u_{n} /\left\|u_{n}\right\|$, $n \geq 1$. Then $\left\|y_{n}\right\|=1$ for all $n \geq 1$ and so we may assume that

$$
y_{n} \stackrel{\mathrm{w}}{\longrightarrow} y \quad \text { in } W_{0}^{1, p}(\Omega) \quad \text { and } \quad y_{n} \rightarrow y \quad \text { in } L^{p}(\Omega) \text { as } n \rightarrow \infty .
$$

From (3.14) we have

$$
\begin{aligned}
\left|\left\langle A_{p}\left(y_{n}\right), h\right\rangle+\frac{1}{\left\|u_{n}\right\|^{p-2}}\left\langle A\left(y_{n}\right), h\right\rangle-\int_{\Omega} \frac{f\left(z, u_{n}\right)}{\left\|u_{n}\right\|^{p-1}} h d z\right| & \\
\leq & \frac{\varepsilon_{n}\|h\|}{\left\|u_{n}\right\|^{p-1}\left(1+\left\|u_{n}\right\|\right)}
\end{aligned}
$$

for all $h \in W_{0}^{1, p}(\Omega)$ and with $\varepsilon_{n} \rightarrow 0^{+}$. Hypothesis $\left(\mathrm{H}_{1}\right)$ (i) implies that $\left\{N_{f}\left(u_{n}\right) /\left\|u_{n}\right\|^{p-1}\right\}_{n \geq 1} \subseteq L^{p^{\prime}}(\Omega)$ is bounded. So, if in (3.20) we choose $h=$ $y_{n}-y \in W_{0}^{1, p}(\Omega)$, pass to the limit as $n \rightarrow \infty$ and use (3.19), then

$$
\lim _{n \rightarrow \infty}\left\langle A_{p}\left(y_{n}\right), y_{n}-y\right\rangle=0(\text { recall } p>2) \Rightarrow y_{n} \rightarrow y \quad \text { in } W_{0}^{1, p}(\Omega),
$$

hence $\|y\|=1$. From (3.18), we have

$$
\begin{aligned}
\eta_{1}\left\|y_{n}\right\|_{\tau}^{\tau} \leq \frac{M_{4}}{\left\|u_{n}\right\|^{\tau}}+\frac{c_{2}}{\left\|u_{n}\right\|^{\tau-2}} \quad \text { with } c_{2}=\left(\frac{p}{2}-1\right)>0 & (\text { recall } p>2) \\
& \Rightarrow \eta_{1}\|y\|_{\tau}^{\tau} \leq 0,
\end{aligned}
$$

hence $y=0$, contradicting (3.21). This proves Claim.

By virtue of Claim, we may assume that

$$
u_{n} \stackrel{\mathrm{w}}{\longrightarrow} u \quad \text { in } W_{0}^{1, p}(\Omega) \quad \text { and } \quad u_{n} \rightarrow u \quad \text { in } L^{p}(\Omega) \text { as } n \rightarrow \infty .
$$

In (3.14) we choose $h=u_{n}-u \in W_{0}^{1, p}(\Omega)$, pass to the limit as $n \rightarrow \infty$ and use (3.22). Then

$$
\begin{aligned}
& \lim _{n \rightarrow \infty}\left[\left\langle A_{p}\left(u_{n}\right), u_{n}-u\right\rangle+\left\langle A\left(u_{n}\right), u_{n}-u\right\rangle\right]=0, \\
& \quad \Rightarrow \limsup _{n \rightarrow \infty}\left[\left\langle A_{p}\left(u_{n}\right), u_{n}-u\right\rangle+\left\langle A(u), u_{n}-u\right\rangle\right] \leq 0 \quad \text { (since } A \text { is monotone) } \\
& \quad \Rightarrow \limsup _{n \rightarrow \infty}\left\langle A_{p}\left(u_{n}\right), u_{n}-u\right\rangle \leq 0 \\
& \Rightarrow u_{n} \rightarrow u \quad \text { in } W_{0}^{1, p}(\Omega)
\end{aligned}
$$


Therefore $\varphi$ satisfies the C-condition.

Proposition 3.5. If hypotheses $\left(\mathrm{H}_{1}\right)(\mathrm{i})-(\mathrm{iv})$ hold, then $u=0$ is a local minimizer for the functionals $\varphi_{ \pm}$and $\varphi$.

Proof. We do the proof for the functional $\varphi_{+}$, the proofs for $\varphi_{-}$and $\varphi$ being similar. By virtue of hypotheses $\left(\mathrm{H}_{1}\right)(\mathrm{i})$,(iv) given $\varepsilon>0$ we can find $c_{3}=c_{3}(\varepsilon)>0$ such that

$$
F(z, x) \leq \frac{1}{2}(\vartheta(z)+\varepsilon) x^{2}+c_{3}|x|^{p} \quad \text { for a.a. } z \in \Omega, \text { all } x \in \mathbb{R} .
$$

Then, for all $u \in W_{0}^{1, p}(\Omega)$, we have

$$
\begin{aligned}
& \varphi_{+}(u)=\frac{1}{p}\|D u\|_{p}^{p}+\frac{1}{2}\|D u\|_{2}^{2}-\int_{\Omega} F_{+}(z, u) d z \\
& \geq \frac{1}{p}\|D u\|_{p}^{p}+\frac{1}{2}\|D u\|_{2}^{2}-\frac{1}{2} \int_{\Omega}(\vartheta(z)+\varepsilon)\left(u^{+}\right)^{2} d z-c_{3}\left\|u^{+}\right\|_{p}^{p} \\
& \text { (see (3.23)) } \\
& \geq \frac{1}{2}\left[\left(\|D u\|_{2}^{2}-\int_{\Omega} \vartheta(z) u^{2} d z\right)-\frac{\varepsilon}{\widehat{\lambda}_{1}(2)}\|D u\|_{2}^{2}\right]-c_{4}\|u\|^{p} \\
& \text { for some } c_{4}>0 \text { (see (2.3)) } \\
& \geq \frac{1}{2}\left[c_{5}-\frac{\varepsilon}{\widehat{\lambda}_{1}(2)}\right]\|u\|^{2}-c_{4}\|u\|^{p} \quad \text { for some } c_{5}>0
\end{aligned}
$$

(see Papageorgiou and Kyritsi [20, p. 356]). Choosing $\varepsilon \in\left(0, \widehat{\lambda}_{1}(2) c_{5}\right)$, we infer that

$$
\varphi_{+}(u) \geq c_{6}\|u\|^{2}-c_{4}\|u\|^{p} \quad \text { for some } c_{6}>0 .
$$

Since $p>2$, from (3.24) we see that for $\rho \in(0,1)$ small, we have

$$
\begin{aligned}
\varphi_{+}(u) \geq 0=\varphi_{+}(0) \quad \text { for all } u \in W_{0}^{1, p}(\Omega) \text { with }\|u\| \leq \rho, \\
\quad \Rightarrow u=0 \text { is a local minimizer for the functional } \varphi_{+} .
\end{aligned}
$$

Similarly we show that $u=0$ is also a local minimizer for the functionals $\varphi_{-}$ and $\varphi$.

Now we are ready to produce constant sign solutions.

Theorem 3.6. If hypotheses $\left(\mathrm{H}_{1}\right)$ hold, then problem (1.1) admits at least two nontrivial constant sign solutions

$$
u_{0} \in \operatorname{int} C_{+} \quad \text { and } \quad v_{0} \in-\operatorname{int} C_{+} .
$$

Proof. First we produce a positive solution. From Proposition 3.5 we see that we can find $\rho \in(0,1)$ small such that

$$
\varphi_{+}(0)=0<\inf \left[\varphi_{+}(u):\|u\|=\rho\right]=m_{\rho}^{+}
$$


(see Aizicovici, Papageorgiou and Staicu [1], proof of Proposition 29). By virtue of hypotheses $\left(\mathrm{H}_{1}\right)(\mathrm{i}),(\mathrm{ii})$, given $\varepsilon>0$, we can find $c_{7}=c_{7}(\varepsilon)>0$ such that

$$
F(z, x) \geq \frac{1}{p}\left(\widehat{\lambda}_{m}(p)-\varepsilon\right)|x|^{p}-c_{7} \quad \text { for a.a. } z \in \Omega \text {, all } x \in \mathbb{R} .
$$

Then, for $t>0$, we have

$$
\begin{aligned}
\varphi_{+}\left(t \widehat{u}_{1}(p)\right) & =\frac{t^{p}}{p} \widehat{\lambda}_{1}(p)+\frac{t^{2}}{2}\left\|D \widehat{u}_{1}(p)\right\|_{2}^{2}=\int_{\Omega} F\left(z, t \widehat{u}_{1}\right) d z \\
& \leq \frac{t^{p}}{p}\left[\widehat{\lambda}_{1}(p)-\widehat{\lambda}_{m}(p)+\varepsilon\right]+\frac{t^{2}}{2}\left\|D \widehat{u}_{1}(p)\right\|_{2}^{2}+c_{7}|\Omega|_{N}
\end{aligned}
$$

(see (3.26)). Choose $\varepsilon \in\left(0, \widehat{\lambda}_{m}(p)-\widehat{\lambda}_{1}(p)\right)$ (recall that $\left.m \geq 2\right)$. Then

$$
\begin{aligned}
\varphi_{+}\left(t \widehat{u}_{1}(p)\right) & \leq-\frac{c_{8} t^{p}}{p}+\frac{t^{2}}{2}\left\|D \widehat{u}_{1}(p)\right\|_{2}^{2}+c_{7}|\Omega|_{N} \quad\left(\text { for some } c_{8}>0\right) \\
& \Rightarrow \frac{\varphi_{+}\left(t \widehat{u}_{1}(p)\right)}{t^{p}} \leq-\frac{c_{8}}{p}+\frac{1}{2 t^{p-2}}\left\|D \widehat{u}_{1}(p)\right\|_{2}^{2}+\frac{c_{7}}{t^{p}}|\Omega|_{N} \\
& \Rightarrow \limsup _{t \rightarrow+\infty} \frac{\varphi_{+}\left(t \widehat{u}_{1}(p)\right)}{t^{p}} \leq-\frac{c_{8}}{p}<0 \quad(\text { recall } p>2) \\
& \Rightarrow \lim _{t \rightarrow+\infty} \varphi_{+}\left(t \widehat{u}_{1}(p)\right)=-\infty .
\end{aligned}
$$

From (3.25), (3.27) and Proposition 3.3, we see that we can apply Theorem 2.1 (the mountain pass theorem) and find $u_{0} \in W_{0}^{1, p}(\Omega)$ such that

$$
u_{0} \in K_{\varphi_{+}} \text {and } m_{\rho}^{+} \leq \varphi_{+}\left(u_{0}\right) .
$$

From (3.25) and (3.28) it follows that $u_{0} \neq 0$. Also

$$
\varphi_{+}^{\prime}\left(u_{0}\right)=0(\operatorname{see}(3.28)) \quad \Rightarrow \quad A_{p}\left(u_{0}\right)+A\left(u_{0}\right)=N_{f_{+}}\left(u_{0}\right) .
$$

On (3.29) we act with $-u_{0}^{-} \in W_{0}^{1, p}(\Omega)$ and obtain

$$
\left\|D u_{0}^{-}\right\|_{p}^{p}+\left\|D u_{0}^{-}\right\|_{2}^{2}=0 \quad \Rightarrow \quad u_{0} \geq 0, u_{0} \neq 0 .
$$

Then from (3.29) we see that $u_{0}$ is a nontrivial solution of (1.1). From Ladyzhenskaya and Uraltseva [18, p. 286], we have $u_{0} \in L^{\infty}(\Omega)$. Then we can apply Theorem 1 of Lieberman [19] and conclude that $u_{0} \in C_{+} \backslash\{0\}$. Let $\rho=\left\|u_{0}\right\|_{\infty}$ and let $\xi_{\rho}>0$ be as postulated by hypothesis $\left(\mathrm{H}_{1}\right)(\mathrm{v})$. Then

$$
\begin{array}{rc}
-\Delta_{p} u_{0}(z)-\Delta u_{0}(z)+\xi_{\rho} u_{0}(z)^{p-1}=f\left(z, u_{0}(z)\right)+\xi_{\rho} u_{0}(z)^{p-1} \geq 0 \\
& \text { for a.a. } z \in \Omega \\
\Rightarrow \Delta_{p} u_{0}(z)+\Delta u_{0}(z) \leq \xi_{\rho} u_{0}(z)^{p-1} & \text { for a.a. } z \in \Omega .
\end{array}
$$

Then from (3.30) and Pucci and Serrin [23, pp.111,120], we infer that $u_{0} \in$ $\operatorname{int} C_{+}$.

Similarly, working with the functional $\varphi_{-}$, via the mountain pass theorem, we produce $v_{0} \in-\operatorname{int} C_{+}$a negative solution of problem (1.1). 
To produce a third nontrivial solution, we need to strengthen our hypotheses on the reaction $f(z, \cdot)$ and employ also tools from the Morse theory. The new hypotheses on $f$, are the following:

$\left(\mathrm{H}_{2}\right) \quad f: \Omega \times \mathbb{R} \rightarrow \mathbb{R}$ is a measurable function such that, for almost all $z \in \Omega$ $f(z, 0)=0, f(z, \cdot) \in C^{1}(\mathbb{R})$ and

(i) $\left|f_{x}^{\prime}(z, x)\right| \leq a(z)\left(1+|x|^{p-2}\right)$ for almost all $z \in \Omega$, all $x \in \mathbb{R}$ and with $a \in L^{\infty}(\Omega)_{+}$;

(ii) $\lim _{x \rightarrow \pm \infty} \frac{f(z, x)}{|x|^{p-2} x}=\widehat{\lambda}_{m}(p)$ uniformly for almost all $z \in \Omega$ and with $m \geq 2$;

(iii) if $F(z, x)=\int_{0}^{x} f(z, s) d s$, then there exists $\tau \in(2, p)$ such that

$\lim _{x \rightarrow \pm \infty} \frac{p F(z, x)-f(z, x) x}{|x|^{\tau}}=+\infty \quad$ uniformly for a.a. $z \in \Omega$;

(iv) $f_{x}^{\prime}(z, 0)=\lim _{x \rightarrow 0} \frac{f(z, x)}{x}$ uniformly for almost all $z \in \Omega$ and

$$
f_{x}^{\prime}(z, 0) \leq \widehat{\lambda}_{1}(2) \quad \text { for a.a. } z \in \Omega, \quad f_{x}^{\prime}(\cdot, 0) \not \equiv \widehat{\lambda}_{1}(2) ;
$$

(v) for every $\rho>0$, there exists $\xi_{\rho}>0$ such that

$$
f(z, x) x+\xi_{\rho}|x|^{p} \geq 0 \quad \text { for a.a. } z \in \Omega \text {, all }|x| \leq \rho .
$$

Under these stronger conditions on the reaction, we can produce a third nontrivial solution and have the following multiplicity theorem.

THEOREM 3.7. If hypotheses $\left(\mathrm{H}_{2}\right)$ hold, then problem (1.1) has at least three nontrivial solutions

$$
u_{0} \in \operatorname{int} C_{+}, \quad v_{0} \in-\operatorname{int} C_{+} \quad \text { and } \quad y_{0} \in C_{0}^{1}(\bar{\Omega}) \backslash\{0\} .
$$

Proof. The two nontrivial constant sign solutions $u_{0} \in \operatorname{int} C_{+}$and $v_{0} \in$ - int $C_{+}$are guaranteed by Theorem 3.6. From the proof of Theorem 3.6, we know that $u_{0} \in \operatorname{int} C_{+}$is a critical point of mountain pass type for the functional $\varphi_{+}$, while $v_{0} \in-\operatorname{int} C_{+}$is a critical point of mountain pass type for the functional $\varphi_{-}$. Therefore we have

$$
C_{1}\left(\varphi_{+}, u_{0}\right) \neq 0 \quad \text { and } \quad C_{1}\left(\varphi_{-}, v_{0}\right) \neq 0 .
$$

We consider the homotopy

$$
h(t, u)=(1-t) \varphi_{+}(u)+t \varphi(u) \quad \text { for all }(t, u) \in[0,1] \times W_{0}^{1, p}(\Omega) .
$$

We assume that $K_{\varphi}$ is finite (otherwise we already have infinitely many distinct solutions for problem (1.1)). Suppose that we can find $\left\{t_{n}\right\}_{n \geq 1} \subseteq[0,1]$ and $\left\{u_{n}\right\}_{n \geq 1} \subseteq W_{0}^{1, p}(\Omega)$ such that

$$
t_{n} \rightarrow t, u_{n} \rightarrow u_{0} \quad \text { in } W_{0}^{1, p}(\Omega) \quad \text { and } \quad h_{u}^{\prime}\left(t_{n}, u_{n}\right)=0 \quad \text { for all } n \geq 1 .
$$


From (3.32), we have

$$
\begin{aligned}
A_{p}\left(u_{n}\right)+A\left(u_{n}\right)=\left(1-t_{n}\right) N_{f_{+}}\left(u_{n}\right)+t_{n} N_{f}\left(u_{n}\right) & \text { for all } n \geq 1 \\
\Rightarrow-\Delta_{p} u_{n}(z)-\Delta u_{n}(z)=\left(1-t_{n}\right) f_{+}\left(z, u_{n}(z)\right)+t_{n} f\left(z, u_{n}(z)\right) & \text { for a.a. } z \in \Omega, \text { all } n \geq 1 .
\end{aligned}
$$

From Ladyzhenskaya and Uraltseva [18, p. 286], we know that we can find $M_{5}>0$ such that $\left\|u_{n}\right\|_{\infty} \leq M_{5}$ for all $n \geq 1$ (see also (3.32)). Then Theorem 1 of Lieberman [19] implies that there exist $\alpha \in(0,1)$ and $M_{6}>0$ such that

$$
u_{n} \in C_{0}^{1, \alpha}(\bar{\Omega}) \quad \text { and } \quad\left\|u_{n}\right\|_{C_{0}^{1, \alpha}(\bar{\Omega})} \leq M_{6} \quad \text { for all } n \geq 1
$$

From (3.33) and the compact embedding of $C_{0}^{1, \alpha}(\bar{\Omega})$ into $C_{0}^{1}(\bar{\Omega})$, we have $u_{n} \rightarrow$ $u_{0}$ in $C_{0}^{1}(\bar{\Omega})$ (see $(3.32)$ ). Since $u_{0} \in \operatorname{int} C_{+}$(see Theorem 3.6), we infer that $u_{n} \in \operatorname{int} C_{+}$for all $n \geq 1$ and so $\left\{u_{n}\right\}_{n \geq n_{0}}$ is a sequence of distinct positive solutions of problem (1.1), a contradiction to our hypothesis that $K_{\varphi}$ is finite. So, (3.32) cannot occur and then the homotopy invariance of critical groups, implies that

$$
C_{k}\left(\varphi_{+}, u_{0}\right)=C_{k}\left(\varphi, u_{0}\right) \text { for all } k \geq 0 .
$$

Similarly we show that

$$
C_{k}\left(\varphi_{-}, v_{0}\right)=C_{k}\left(\varphi, v_{0}\right) \quad \text { for all } k \geq 0 \quad\left(\text { recall } v_{0} \in-\operatorname{int} C_{+}\right) .
$$

From (3.31), (3.34) and (3.35), we have

$$
C_{1}\left(\varphi, u_{0}\right) \neq 0, \quad C_{1}\left(\varphi, v_{0}\right) \neq 0 .
$$

Since $\varphi \in C^{2}\left(W_{0}^{1, p}(\Omega)\right.$ ) (see hypotheses $\left(\mathrm{H}_{2}\right)$ ), from (3.36) and Papageorgiou and Smyrlis [22] (see also Papageorgiou and Rădulescu [21]), it follows that

$$
C_{k}\left(\varphi, u_{0}\right)=C_{k}\left(\varphi, v_{0}\right)=\delta_{k, 1} \mathbb{Z} \text { for all } k \geq 0 .
$$

Also, Proposition 3.5 implies that

$$
C_{k}(\varphi, 0)=\delta_{k, 0} \mathbb{Z} \text { for all } k \geq 0 .
$$

Hypothesis $\left(\mathrm{H}_{2}\right)$ (iii) implies that given $\xi>0$, we can find $M_{7}=M_{7}(\xi)>0$ such that

$$
p F(z, x)-f(z, x) x \geq \xi|x|^{\tau} \quad \text { for a.a. } z \in \Omega \text {, all }|x| \geq M_{7} .
$$

For almost all $z \in \Omega$ and for $s \neq 0$, we have

$$
\begin{aligned}
\frac{d}{d s} \frac{F(z, s)}{|s|^{p}} & =\frac{f(z, s)|s|^{p}-p|s|^{p-2} s F(z, s)}{|s|^{2 p}} \\
& \leq \frac{|s|^{p-2} s[f(z, s) s-p F(z, s)]}{|s|^{2 p}}=\frac{f(z, s) s-p F(z, s)}{|s|^{p} s}
\end{aligned}
$$


Then for $s>0$, we have

$$
\begin{aligned}
& \text { (3.40) } \frac{d}{d s} \frac{F(z, s)}{s^{p}}=\frac{f(z, s) s-p F(z, s)}{s^{p+1}} \leq-\xi s^{\tau-p-1} \\
& \quad \text { for a.a. } z \in \Omega \text {, all } s \geq M_{7} \text { (see (3.39)) } \\
& \Rightarrow \frac{F(z, y)}{y^{p}}-\frac{F(z, x)}{x^{p}} \leq \frac{\xi}{p-\tau}\left[\frac{1}{y^{p-\tau}}-\frac{1}{x^{p-\tau}}\right] \quad \text { for a.a. } z \in \Omega \text {, all } y \geq x \geq M_{7} .
\end{aligned}
$$

Note that hypothesis $\left(\mathrm{H}_{2}\right)$ (ii) implies that

$$
\lim _{s \rightarrow \pm \infty} \frac{p F(z, s)}{|s|^{p}}=\widehat{\lambda}_{m}(p) \quad \text { uniformly for a.a. } z \in \Omega .
$$

Recall that $\tau<p$ (see hypothesis $\left(\mathrm{H}_{2}\right)$ (iii)). So, if in (3.40) we let $y \rightarrow+\infty$ and use (3.41), then

$$
\begin{aligned}
& \frac{\widehat{\lambda}_{m}(p)}{p}-\frac{F(z, x)}{x^{p}} \leq-\frac{\xi}{p-\tau} \frac{1}{x^{p-\tau}} \quad \text { for a.a. } z \in \Omega, \text { all } x \geq M_{7} \\
& \Rightarrow \frac{\widehat{\lambda}_{m}(p)}{p} x^{p}-F(z, x) \leq-\frac{\xi}{p-\tau} x^{\tau} \quad \text { for a.a. } z \in \Omega, \text { all } x \geq M_{7} \\
& \Rightarrow \frac{\widehat{\lambda}_{m}(p) x^{p}-p F(z, x)}{x^{\tau}} \leq-\xi \frac{p}{p-\tau} \quad \text { for a.a. } z \in \Omega, \text { all } x \geq M_{7} .
\end{aligned}
$$

Since $\xi>0$ is arbitrary, it follows that

$$
\lim _{x \rightarrow+\infty} \frac{\widehat{\lambda}_{m}(p) x^{p}-p F(z, x)}{x^{\tau}}=-\infty \quad \text { uniformly for a.a. } z \in \Omega .
$$

For $s<0$, we have

$$
\begin{aligned}
\frac{d}{d s} \frac{F(z, s)}{|s|^{p}}= & \frac{f(z, s) s-p F(z, s)}{|s|^{p} s} \geq-\frac{\xi}{|s|^{p-\tau} s} \\
& \text { for a.a. } z \in \Omega, \text { all } s \leq-M_{7}(\text { see }(3.39) \text { and recall that } s<0) \\
& \Rightarrow \frac{F(z, x)}{|x|^{p}}-\frac{F(z, y)}{|y|^{p}} \geq-\frac{\xi}{p-\tau}\left[\frac{1}{|y|^{p-\tau}}-\frac{1}{|x|^{p-\tau}}\right] \\
& \quad \text { for a.a. } z \in \Omega, \text { all } y \leq x \leq-M_{7} .
\end{aligned}
$$

Letting $y \rightarrow-\infty$ and using (3.41), we obtain

$$
\begin{array}{r}
\frac{F(z, x)}{|x|^{p}}-\frac{\hat{\lambda}_{m}(p)}{p} \geq \frac{\xi}{p-\tau} \frac{1}{|x|^{p-\tau}} \quad \text { for a.a. } z \in \Omega \text {, all } x \leq-M_{7} \text { (see (3.41)) } \\
\Rightarrow \frac{\widehat{\lambda}_{m}(p)|x|^{p}-p F(z, x)}{|x|^{\tau}} \leq-\xi \frac{p}{p-\tau} \quad \text { for a.a. } z \in \Omega \text {, all } x \leq-M_{7} .
\end{array}
$$

Since $\xi>0$ is arbitrary, we infer that

$$
\lim _{x \rightarrow-\infty} \frac{\widehat{\lambda}_{m}(p)|x|^{p}-p F(z, x)}{|x|^{\tau}}=-\infty \quad \text { uniformly for a.a. } z \in \Omega \text {. }
$$


Therefore we have proved that

$$
\lim _{x \rightarrow \pm \infty} \frac{\widehat{\lambda}_{m}(p)|x|^{p}-p F(z, x)}{|x|^{\tau}}=-\infty \quad \text { uniformly for a.a. } z \in \Omega .
$$

We introduce the following two sets:

$$
\begin{aligned}
S_{r} & =\left\{u \in W_{0}^{1, p}(\Omega):\|u\|^{p}=r^{p},\|D u\|_{p}^{p} \leq \widehat{\lambda}_{m}(p)\|u\|_{p}^{p}\right\}, \\
A & =\left\{u \in W_{0}^{1, p}(\Omega):\|D u\|_{p}^{p} \geq \widehat{\lambda}_{m+1}(p)\|u\|_{p}^{p}\right\} .
\end{aligned}
$$

Note that $S_{r}$ is a $C^{1}$-Banach manifold with boundary.

From Degiovanni and Lancelotti [9, Theorem 3.2], we have

$$
\operatorname{ind}\left(S_{r}\right)=\operatorname{ind}\left(W_{0}^{1, p}(\Omega) \backslash A\right)=m .
$$

Also, from Cingolani and Degiovanni [7, Theorem 3.6] we know that the sets $S$ and $A$ homologically link in dimension $m$.

Hypotheses $\left(\mathrm{H}_{2}\right)(\mathrm{i})$, (ii) imply that given $\varepsilon>0$, we can find $c_{9}=c_{9}(\varepsilon)>0$ such that

$$
F(z, x) \leq \frac{\widehat{\lambda}_{m}(p)+\varepsilon}{p}|x|^{p}+c_{9} \quad \text { for a.a. } z \in \Omega, \text { all } x \in \mathbb{R} .
$$

Recall that $\widehat{\lambda}_{m}(p)<\widehat{\lambda}_{m+1}(p)$. Then, for all $u \in A$, we have

$$
\varphi(u) \geq \frac{\widehat{\lambda}_{m+1}(p)-\widehat{\lambda}_{m}(p)-\varepsilon}{p}\|u\|_{p}^{p}-c_{9}|\Omega|_{N} .
$$

Choosing $\varepsilon \in\left(0, \widehat{\lambda}_{m+1}(p)-\widehat{\lambda}_{m}(p)\right)$, we obtain $\inf _{A} \varphi>-\infty$. Also, we claim that $\sup _{S_{r}} \varphi<\inf _{A} \varphi$ for $r>0$ big enough. Arguing by contradiction, suppose that we can find $\left\{u_{n}\right\}_{n \geq 1} \subseteq S$ and $\xi>0$ such that

$$
-\xi \leq \varphi\left(u_{n}\right) \text { for all } n \geq 1 \text { and }\left\|u_{n}\right\| \rightarrow+\infty \text {. }
$$

Let $y_{n}=u_{n} /\left\|u_{n}\right\|, n \geq 1$. Then $\left\|y_{n}\right\|=1$ for all $n \geq 1$. So, we may assume that $y_{n} \stackrel{\mathrm{w}}{\longrightarrow} y \quad$ in $W_{0}^{1, p}(\Omega) \quad$ and $\quad y_{n} \rightarrow y \quad$ in $L^{p}(\Omega)$ as $n \rightarrow \infty$.

We have

$$
\begin{array}{rlrl}
1= & \left\|D y_{n}\right\|_{p}^{p} \leq \widehat{\lambda}_{m}(p)\left\|y_{n}\right\|_{p}^{p} & & \left(\text { recall } u_{n} \in S \text { for all } n \geq 1\right) \\
& \Rightarrow 1 \leq \widehat{\lambda}_{m}(p)\|y\|_{p}^{p} & & (\text { see }(3.42)) \\
& \Rightarrow y \neq 0 . &
\end{array}
$$

Therefore $\left|u_{n}(z)\right| \rightarrow+\infty$ for almost all $z \in \Omega_{0}=\{z \in \Omega: y(z) \neq 0\}$ and $\left|\Omega_{0}\right|_{N}>0$. We have (see (3.43))

$$
-\frac{p \xi}{\left\|u_{n}\right\|^{\tau}} \leq \int_{\Omega} \frac{\widehat{\lambda}_{m}(p)\left|u_{n}\right|^{p}-p F\left(z, u_{n}\right)}{\left|u_{n}\right|^{\tau}}\left|y_{n}\right|^{\tau} d z+\frac{p}{2} \frac{1}{\left\|u_{n}\right\|^{\tau-2}}\left\|D y_{n}\right\|_{2}^{2}
$$

Since $\tau>2$, passing to the limit as $n \rightarrow \infty$ and since $\left\|u_{n}\right\| \rightarrow \infty$, we reach a contradiction to (3.42). This proves the claim, namely that $\varphi(u) \rightarrow-\infty$ as 
$\|u\| \rightarrow+\infty$ with $u \in S$. Therefore choosing $r>0$ big enough, we can have $\sup _{S} \varphi<\inf _{A} \varphi$. So, we can apply Theorem 3.2 of Cingolani and Degiovanni [7] and infer that there exists $y_{0} \in W_{0}^{1, p}(\Omega)$ such that $y_{0} \in K_{\varphi}$ and $C_{m}\left(\varphi, y_{0}\right) \neq$ 0 . Comparing with (3.37) and (3.38), we obtain $y_{0} \notin\left\{0, u_{0}, v_{0}\right\}$. Also, since $y_{0} \in K_{\varphi}, y_{0}$ is a nontrivial solution of problem (1.1) and as before the nonlinear regularity theory implies that $y_{0} \in C_{0}^{1}(\bar{\Omega})$.

REMARK 3.8. Is it possible to show that $y_{0}$ is nodal?

\section{Superlinear problems}

In this section, we assume that the reaction $f(z, \cdot)$ is $(p-1)$-superlinear near $\pm \infty$ without satisfying the AR-condition and has $z$-dependent zeros of constant sign. For such problems, we prove a multiplicity theorem producing six nontrivial solutions all with sign information and a second multiplicity theorem producing five solutions.

The hypotheses on the reaction $f$ are the following:

$\left(\mathrm{H}_{3}\right) f: \Omega \times \mathbb{R} \rightarrow \mathbb{R}$ is a measurable function such that for almost all $z \in \Omega$, $f(z, 0)=0, f(z, \cdot) \in C^{1}(\mathbb{R})$ and

(i) $\left|f_{x}^{\prime}(z, x)\right| \leq a(z)\left(1+|x|^{r-2}\right)$ for almost all $z \in \Omega$, all $x \in \mathbb{R}$, with $a \in L^{\infty}(\Omega)_{+}$and $r \in\left(p, p^{*}\right)$;

(ii) $\lim _{x \rightarrow \pm \infty} \frac{F(z, x)}{|x|^{p}}=+\infty$ uniformly for almost all $z \in \Omega$;

(iii) there exist $\tau \in(\max \{1,(r-p) N / p\}, r)$ and $\eta_{0}>0$ such that

$\eta_{0} \leq \liminf _{x \rightarrow \pm \infty} \frac{f(z, x) x-p F(z, x)}{|x|^{\tau}}$ uniformly for almost all $z \in \Omega$;

(iv) $f_{x}^{\prime}(z, 0)=\lim _{x \rightarrow 0} \frac{f(z, x)}{x}$ uniformly for almost all $z \in \Omega$ and for some $m \geq 2$, we have

$$
\begin{gathered}
f_{x}^{\prime}(z, 0) \in\left[\widehat{\lambda}_{m}(2), \widehat{\lambda}_{m+1}(2)\right] \quad \text { for almost all } z \in \Omega, \\
f_{x}^{\prime}(\cdot, 0) \neq \widehat{\lambda}_{m}(2), \quad f_{x}^{\prime}(\cdot, 0) \neq \widehat{\lambda}_{m+1}(2)
\end{gathered}
$$

(v) there exist functions $w_{ \pm} \in W^{1, p}(\Omega) \cap C(\bar{\Omega})$ such that

$$
\begin{array}{ll}
w_{-}(z) \leq c_{-}<0<c_{+} \leq w_{+}(z) & \text { for all } z \in \bar{\Omega}, \\
f\left(z, w_{+}(z)\right) \leq 0 \leq f\left(z, w_{-}(z)\right) & \text { for a.a. } z \in \Omega,
\end{array}
$$

and

$$
\begin{array}{r}
\left\langle A_{p}\left(w_{-}\right), \vartheta\right\rangle+\left\langle A\left(w_{-}\right), \vartheta\right\rangle \leq 0 \leq\left\langle A_{p}\left(w_{+}\right), \vartheta\right\rangle+\left\langle A\left(w_{+}\right), \vartheta\right\rangle \\
\quad \text { for all } \vartheta \in W^{1, p}(\Omega) \text { with } \vartheta \geq 0 ;
\end{array}
$$


(vi) for every $\rho>0$, there exists $\xi_{\rho}>0$ such that for almost all $z \in \Omega$ the map $x \rightarrow f(z, x)+\xi_{\rho}|x|^{p-2} x$ is nondecreasing on $[-\rho, \rho]$.

REMARK 4.1. We stress that now the regularity condition on $f(z, \cdot)$ is stronger, namely $f(z, \cdot) \in C^{1}(\mathbb{R})$. Hypothesis $\left(\mathrm{H}_{3}\right)$ (ii) implies that the potential $F(z, \cdot)$ is $p$-superlinear near $\pm \infty$. This hypothesis together with $\left(\mathrm{H}_{3}\right)$ (iii) imply that the reaction $f(z, \cdot)$ is $(p-1)$-superlinear near $\pm \infty$. Note that, unlike most works on superlinear problems, we do not employ the AR-condition. We recall that this condition says that there exist $q>p$ and $M>0$ such that

$$
\begin{aligned}
& 0<q F(z, x) \leq f(z, x) x \quad \text { for a.a. } z \in \Omega, \text { all }|x| \geq M, \\
& 0<\underset{\Omega}{\operatorname{essinf}} F(\cdot, \pm M) .
\end{aligned}
$$

The AR-condition implies the following weaker unilateral growth condition:

$$
c_{10}|x|^{q} \leq F(z, x) \quad \text { for a.a. } z \in \Omega \text {, all }|x| \geq M \text { and some } c_{10}>0 .
$$

From this, we have the much weaker condition

$$
\lim _{x \rightarrow \pm \infty} \frac{F(z, x)}{|x|^{p}}=+\infty \quad \text { uniformly for a.a. } z \in \Omega .
$$

Here we employ the above asymptotic condition and hypothesis $\left(\mathrm{H}_{3}\right)$ (iii), which together are weaker than the AR-condition and incorporate in our framework superlinear reactions with "slower" growth near $\pm \infty$ which fail to satisfy the AR-condition (see the example below). Hypothesis $\left(\mathrm{H}_{3}\right)$ (iv) implies that asymptotically at 0 we have nonuniform nonresonance with respect to the spectral interval $\left[\widehat{\lambda}_{m}(2), \widehat{\lambda}_{m+1}(2)\right]$. Finally hypothesis $\left(\mathrm{H}_{3}\right)(\mathrm{v})$ implies the existence of $z$-dependent zeros of constant sign. Evidently this hypothesis is satisfied if there exist $c_{-}<0<c_{+}$such that

$$
f\left(z, c_{+}\right) \leq 0 \leq f\left(z, c_{-}\right) \quad \text { for a.a. } z \in \Omega .
$$

As a final remark concerning hypotheses $\left(\mathrm{H}_{3}\right)$, we mention that by virtue of hypotheses $\left(\mathrm{H}_{3}\right)(\mathrm{i}),(\mathrm{iv})$, we can find $\xi_{0}>\widehat{\lambda}_{1}(2)$ and $\xi_{1}>0$ such that

$$
f(z, x) x \geq \xi_{0} x^{2}-\xi_{1}|x|^{r} \quad \text { for a.a. } z \in \Omega \text {, all } x \in \mathbb{R} .
$$

EXAmPLE 4.2. The following function satisfies hypotheses $\left(\mathrm{H}_{3}\right)$. For the sake of simplicity we drop the $z$-dependence

$$
f(x)=|x|^{p-2} x\left(\ln |x|+\frac{1}{p}\right)+\vartheta x-c|x|^{\tau-2} x
$$

with $\tau \in\left(\widehat{\lambda}_{m}, \widehat{\lambda}_{m+1}\right)(m \geq 2), c \geq 1 / p+\vartheta, 2<\tau<p$. Note that this function does not satisfy the AR-condition. 
First we will produce constant sign solutions. As before $\varphi: W_{0}^{1, p}(\Omega) \rightarrow \mathbb{R}$ is the energy functional for problem (1.1) defined by

$$
\varphi(u)=\frac{1}{p}\|D u\|_{p}^{p}+\frac{1}{2}\|D u\|_{2}^{2}-\int_{\Omega} F(z, u(z)) d z \quad \text { for all } u \in W_{0}^{1, p}(\Omega) .
$$

To produce the first two constant sign solutions, the asymptotic condition on $f(z, \cdot)$ at $\pm \infty$ is irrelevant (that is, we do not need hypotheses $\left(\mathrm{H}_{3}\right)$ (ii),(iii)).

Proposition 4.3. If hypotheses $\left(\mathrm{H}_{3}\right)(\mathrm{i})$, (iv)-(vi) hold, then problem (1.1) has two nontrivial constant sign solutions

$$
u_{0} \in \operatorname{int} C_{+} \quad \text { and } \quad v_{0} \in-\operatorname{int} C_{+}
$$

such that $-c_{-} \leq w_{-}(z) \leq v_{0}(z) \leq 0 \leq u_{0}(z) \leq w_{+}(z) \leq c_{+}$for all $z \in \bar{\Omega}$.

Proof. First we produce the positive solution. To this end, we introduce the following truncation of $f(z, \cdot)$ :

$$
\widehat{f}_{+}(z, x)= \begin{cases}0 & \text { if } x<0 \\ f(z, x) & \text { if } 0 \leq x \leq w_{+}(z), \\ f\left(z, w_{+}(z)\right) & \text { if } w_{+}(z)<x\end{cases}
$$

This is a Carathéodory function. Let

$$
\widehat{F}_{+}(z, x)=\int_{0}^{x} \widehat{f}_{+}(z, s) d s
$$

and consider the $C^{1}$-functional $\widehat{\varphi}_{+}: W_{0}^{1, p}(\Omega) \rightarrow \mathbb{R}$ defined by

$$
\widehat{\varphi}_{+}(u)=\frac{1}{p}\|D u\|_{p}^{p}+\frac{1}{2}\|D u\|_{2}^{2}-\int_{\Omega} \widehat{F}_{+}(z, u(z)) d z \quad \text { for all } u \in W_{0}^{1, p}(\Omega) .
$$

It is clear from (4.2) that $\widehat{\varphi}_{+}$is coercive. Also, it is sequentially weakly lower semicontinuous. So, by the Weierstrass theorem, we can find $u_{0} \in W_{0}^{1, p}(\Omega)$ such that

$$
\widehat{\varphi}_{+}\left(u_{0}\right)=\inf \left[\widehat{\varphi}_{+}(u): u \in W_{0}^{1, p}(\Omega)\right]=\widehat{\eta}_{+} .
$$

Hypothesis $\left(\mathrm{H}_{3}\right)(\mathrm{iv})$ implies that, given $\varepsilon>0$, we can find $\delta \in\left(0, c_{+}\right)$such that

$$
\begin{aligned}
f(z, x) \geq & \left(f_{x}^{\prime}(z, 0)-\varepsilon\right) x \quad \text { for a.a. } z \in \Omega, \text { all } x \in[0, \delta] \\
& \Rightarrow F(z, x) \geq \frac{1}{2}\left(f_{x}^{\prime}(z, 0)-\varepsilon\right) x^{2} \quad \text { for a.a. } z \in \Omega, \text { all } x \in[0, \delta] .
\end{aligned}
$$

Let $\widehat{u}_{1}(2) \in \operatorname{int} C_{+}$be the $L^{2}$-normalized principal eigenfunction of $\left(-\Delta, H_{0}^{1}(\Omega)\right)$ and let $t \in(0,1)$ be small such that $t \widehat{u}_{1}(2)(z) \in[0, \delta]$ for all $z \in \bar{\Omega}$. Then

$$
\widehat{\varphi}_{+}\left(t \widehat{u}_{1}(2)\right) \leq \frac{t^{p}}{p}\left\|D \widehat{u}_{1}(2)\right\|_{p}^{p}+\frac{t^{2}}{2}\left[\int_{\Omega}\left(\widehat{\lambda}_{1}(2)-f_{x}^{\prime}(z, 0)\right) \widehat{u}_{1}(2)^{2} d z+\varepsilon\right]
$$


(see (4.4) and recall that $\left.\left\|\widehat{u}_{1}(2)\right\|_{2}=1\right)$. Note that

$$
\xi_{*}=\int_{\Omega}\left[f_{x}^{\prime}(z, 0)-\widehat{\lambda}_{1}(2)\right] \widehat{u}_{1}(2)^{2} d z>0
$$

(see hypothesis $\left.\left(\mathrm{H}_{3}\right)(\mathrm{iv})\right)$. We have

$$
\widehat{\varphi}_{+}\left(t \widehat{u}_{1}(2)\right) \leq \frac{t^{p}}{p}\left\|D \widehat{u}_{1}(2)\right\|_{p}^{p}-\frac{t^{2}}{2}\left[\xi_{*}-\varepsilon\right] .
$$

Choosing $\varepsilon \in\left(0, \xi_{*}\right)$ and since $p>2$, for $t \in(0,1)$ even smaller if necessary, we have

$$
\widehat{\varphi}_{+}\left(t \widehat{u}_{1}(2)\right)<0 \Rightarrow \widehat{\varphi}_{+}\left(u_{0}\right)<0=\widehat{\varphi}_{+}(0)
$$

(see (4.2)), hence $u_{0} \neq 0$. From (4.2), we have

$$
\widehat{\varphi}_{+}^{\prime}\left(u_{0}\right)=0 \quad \Rightarrow \quad A_{p}\left(u_{0}\right)+A\left(u_{0}\right)=N_{\widehat{f}_{+}}\left(u_{0}\right) .
$$

On (4.5) we act with $-u_{0}^{-} \in W_{0}^{1, p}(\Omega)$ and obtain $\left\|D u_{0}^{-}\right\|_{p}^{p}+\left\|D u_{0}^{-}\right\|_{2}^{2}=0$ (see $(4.2))$, hence $u_{0} \geq 0, u_{0} \neq 0$. Also, on (4.4) we act with $\left(u_{0}-w_{+}\right)^{+}$ in $W_{0}^{1, p}(\Omega)$ and obtain

$$
\begin{aligned}
& \left\langle A_{p}\left(u_{0}\right),\left(u_{0}-w_{+}\right)^{+}\right\rangle+\left\langle A\left(u_{0}\right),\left(u_{0}-w_{+}\right)^{+}\right\rangle=\int_{\Omega} \widehat{f}_{+}\left(z, u_{0}\right)\left(u_{0}-w_{+}\right)^{+} d z \\
& =\int_{\Omega} f\left(z, w_{+}\right)\left(u_{0}-w_{+}\right)^{+} d z \quad(\text { see }(4.2)) \\
& \leq\left\langle A_{p}\left(w_{+}\right),\left(u_{0}-w_{+}\right)^{+}\right\rangle+\left\langle A\left(w_{+}\right),\left(u_{0}-w_{+}\right)^{+}\right\rangle \quad\left(\text { see hypothesis }\left(\mathrm{H}_{3}\right)(\mathrm{v})\right) \\
& \Rightarrow\left\langle A_{p}\left(u_{0}\right)-A_{p}\left(w_{+}\right),\left(u_{0}-w_{+}\right)^{+}\right\rangle+\left\langle A\left(u_{0}\right)-A\left(w_{+}\right),\left(u_{0}-w_{+}\right)^{+}\right\rangle \leq 0 \\
& \Rightarrow\left|\left\{u_{0}>w_{+}\right\}\right|_{N}=0,
\end{aligned}
$$

hence $u_{0} \leq w_{+}$. So, we have proved that

$$
u_{0} \in\left[0, w_{+}\right]=\left\{u \in W_{0}^{1, p}(\Omega): 0 \leq u(z) \leq w_{+}(z) \text { a.e. in } \Omega\right\} .
$$

Then from (4.2) and (4.5) it follows that $u_{0}$ is a positive solution of problem (1.1). As before the nonlinear regularity theory (see Lieberman [19]) and the nonlinear maximum principle (see Pucci and Serrin [23, pp. 111, 120]), imply $u_{0} \in \operatorname{int} C_{+}$.

Similarly, we consider the following truncation of $f(z, \cdot)$ :

$$
\widehat{f}_{-}(z, x)= \begin{cases}f\left(z, w_{-}(z)\right) & \text { if } x<w_{-}(z), \\ f(z, x) & \text { if } w_{-}(z) \leq x \leq 0, \\ 0 & \text { if } 0<x\end{cases}
$$

This is a Carathéodory function. We set

$$
\widehat{F}_{-}(z, x)=\int_{0}^{x} \widehat{f}_{-}(z, s) d s
$$


and consider the $C^{1}$-functional $\widehat{\varphi}_{-}: W_{0}^{1, p}(\Omega) \rightarrow \mathbb{R}$ defined by

$$
\widehat{\varphi}_{-}(u)=\frac{1}{p}\|D u\|_{p}^{p}+\frac{1}{2}\|D u\|_{2}^{2}-\int_{\Omega} \widehat{F}_{-}(z, u(z)) d z \quad \text { for all } u \in W_{0}^{1, p}(\Omega) .
$$

Reasoning as above, via the direct method, we produce a negative solution $v_{0} \in$ $-\operatorname{int} C_{+}$with $c_{-} \leq w_{-}(z) \leq v_{0}(z) \leq 0$ for all $z \in \bar{\Omega}$.

Using these two constant sign solutions and the asymptotic conditions at $\pm \infty$ (see hypotheses $\left(\mathrm{H}_{3}\right)$ (ii),(iii)), we can generate two more nontrivial solutions of constant sign.

Proposition 4.4. If hypotheses $\left(\mathrm{H}_{3}\right)$ hold, then problem (1.1) has two additional constant sign solutions

$$
\widehat{u} \in \operatorname{int} C_{+} \quad \text { and } \quad \widehat{v} \in-\operatorname{int} C_{+}
$$

such that $\widehat{u}-u_{0} \in \operatorname{int} C_{+}$and $v_{0}-\widehat{v} \in \operatorname{int} C_{+}$.

Proof. Again, first we show the existence of the second positive solution. To this end, let $u_{0} \in \operatorname{int} C_{+}$be the positive solution produced in Proposition 4.3. We introduce the following truncation of the reaction $f(z, \cdot)$ :

$$
g_{+}(z, x)= \begin{cases}f\left(z, u_{0}(z)\right) & \text { if } x \leq u_{0}(z), \\ f(z, x) & \text { if } u_{0}(z)<x .\end{cases}
$$

This is a Carathéodory function. We set

$$
G_{+}(z, x)=\int_{0}^{x} g_{+}(z, s) d s
$$

and consider the $C^{1}$-functional $\psi_{+}: W_{0}^{1, p}(\Omega) \rightarrow \mathbb{R}$ defined by

$$
\psi_{+}(u)=\frac{1}{p}\|D u\|_{p}^{p}+\frac{1}{2}\|D u\|_{2}^{2}-\int_{\Omega} G_{+}(z, u(z)) d z \quad \text { for all } u \in W_{0}^{1, p}(\Omega) .
$$

From Aizicovici, Papageorgiou and Staicu [2] (proof of Proposition 4), we know that

$$
\psi_{+} \text {satisfies the C-condition. }
$$

Claim. We may assume that $u_{0} \in \operatorname{int} C_{+}$is a local minimizer of $\psi_{+}$.

We consider the following truncation of $g_{+}(z, \cdot)$ (recall that $u_{0} \leq w_{+}$, see Proposition 4.3):

$$
\widehat{g}_{+}(z, x)= \begin{cases}g_{+}(z, x) & \text { if } x \leq w_{+}(z), \\ g_{+}\left(z, w_{+}(z)\right) & \text { if } w_{+}(z)<x .\end{cases}
$$

This is a Carathéodory function. We set

$$
\widehat{G}_{+}(z, x)=\int_{0}^{x} \widehat{g}_{+}(z, s) d s
$$


and consider the $C^{1}$-functional $\widehat{\psi}: W_{0}^{1, p}(\Omega) \rightarrow \mathbb{R}$ defined by

$$
\widehat{\psi}_{+}(u)=\frac{1}{p}\|D u\|_{p}^{p}+\frac{1}{2}\|D u\|_{2}^{2}-\int_{\Omega} \widehat{G}_{+}(z, u(z)) d z \quad \text { for all } u \in W_{0}^{1, p}(\Omega) .
$$

From (4.6) and (4.8) it is clear that $\widehat{\psi}_{+}$is coercive. Also, it is sequentially weakly lower semicontinuous. So, we can find $\widehat{u}_{0} \in W_{0}^{1, p}(\Omega)$ such that

$$
\begin{aligned}
\widehat{\psi}_{+}\left(\widehat{u}_{0}\right)=\inf \left[\widehat{\psi}_{+}(u): u\right. & \left.\in W_{0}^{1, p}(\Omega)\right] \\
& \Rightarrow \widehat{\psi}_{+}^{\prime}\left(\widehat{u}_{0}\right)=0 \Rightarrow A_{p}\left(\widehat{u}_{0}\right)+A\left(\widehat{u}_{0}\right)=N_{\widehat{g}_{+}}\left(\widehat{u}_{0}\right) .
\end{aligned}
$$

On (4.9) first we act with $\left(u_{0}-\widehat{u}_{0}\right)^{+} \in W_{0}^{1, p}(\Omega)$ and obtain

$$
\begin{aligned}
& \left\langle A_{p}\left(\widehat{u}_{0}\right),\left(u_{0}-\widehat{u}_{0}\right)^{+}\right\rangle+\left\langle A\left(\widehat{u}_{0}\right),\left(u_{0}-\widehat{u}_{0}\right)^{+}\right\rangle=\int_{\Omega} \widehat{g}_{+}\left(z, \widehat{u}_{0}\right)\left(u_{0}-\widehat{u}_{0}\right)^{+} d z \\
& =\int_{\Omega} f\left(z, u_{0}\right)\left(u_{0}-\widehat{u}_{0}\right)^{+} d z \quad \quad \text { (see (4.8) and (4.6)) } \\
& =\left\langle A_{p}\left(u_{0}\right),\left(u_{0}-\widehat{u}_{0}\right)^{+}\right\rangle+\left\langle A\left(u_{0}\right),\left(u_{0}-\widehat{u}_{0}\right)^{+}\right\rangle \quad \text { (see Proposition 4.3) } \\
& \Rightarrow\left\langle A_{p}\left(u_{0}\right)-A_{p}\left(\widehat{u}_{0}\right),\left(u_{0}-\widehat{u}_{0}\right)^{+}\right\rangle+\left\langle A\left(u_{0}\right)-A\left(\widehat{u}_{0}\right),\left(u_{0}-\widehat{u}_{0}\right)^{+}\right\rangle=0 \\
& \Rightarrow\left|\left\{u_{0}>\widehat{u}_{0}\right\}\right|_{N}=0 \text {, }
\end{aligned}
$$

hence $u_{0} \leq \widehat{u}_{0}$. Also, on (4.9) we act with $\left(\widehat{u}_{0}-w_{+}\right)^{+} \in W_{0}^{1, p}(\Omega)$. Then

$$
\begin{aligned}
& \left\langle A_{p}\left(\widehat{u}_{0}\right),\left(\widehat{u}_{0}-w_{+}\right)^{+}\right\rangle+\left\langle A\left(\widehat{u}_{0}\right),\left(\widehat{u}_{0}-w_{+}\right)^{+}\right\rangle=\int_{\Omega} \widehat{g}_{+}\left(z, \widehat{u}_{0}\right)\left(\widehat{u}_{0}-w_{+}\right)^{+} d z \\
& \left.=\int_{\Omega} f\left(z, w_{+}\right)\left(\widehat{u}_{0}-w_{+}\right)^{+} d z \quad \text { (see }(4.8) \text { and }(4.6)\right) \\
& \leq\left\langle A_{p}\left(w_{+}\right),\left(\widehat{u}_{0}-w_{+}\right)^{+}\right\rangle+\left\langle A\left(w_{+}\right),\left(\widehat{u}_{0}-w_{+}\right)^{+}\right\rangle \quad\left(\text { see hypothesis }\left(\mathrm{H}_{3}\right)(\mathrm{v})\right) \\
& \Rightarrow\left\langle A_{p}\left(\widehat{u}_{0}\right)-A_{p}\left(w_{+}\right),\left(\widehat{u}_{0}-w_{+}\right)^{+}\right\rangle+\left\langle A\left(\widehat{u}_{0}\right)-A\left(w_{+}\right),\left(\widehat{u}_{0}-w_{+}\right)^{+}\right\rangle \leq 0 \\
& \Rightarrow\left|\left\{\widehat{u}_{0}>w_{+}\right\}\right|_{N}=0,
\end{aligned}
$$

hence $\widehat{u}_{0} \leq w_{+}$. So, we have proved that

$$
\widehat{u}_{0} \in\left[u_{0}, w_{+}\right]=\left\{u \in W_{0}^{1, p}(\Omega): u_{0}(z) \leq u(z) \leq w_{+}(z) \text { a.e. in } \Omega\right\} .
$$

Then from (4.6), (4.8) and (4.9) it follows that $\widehat{u}_{0}$ is a positive solution of problem (1.1) and as before the nonlinear regularity theory and the nonlinear maximum principle imply $\widehat{u}_{0} \in \operatorname{int} C_{+}$.

If $\widehat{u}_{0} \neq u_{0}$, then this is the desired second positive solution of (1.1).

If $\widehat{u}_{0}=u_{0} \in \operatorname{int} C_{+}$, then for $\rho=\left\|w_{+}\right\|_{\infty}$, let $\xi_{\rho}>0$ be as postulated by hypothesis $\left(\mathrm{H}_{3}\right)(\mathrm{vi})$. We have

$$
\begin{aligned}
-\Delta_{p} u_{0}(z)-\Delta u_{0}(z)+\xi_{\rho} u_{0}(z)^{p-1} & =f\left(z, u_{0}(z)\right)+\xi_{\rho} u_{0}(z)^{p-1} \\
& \leq f\left(z, w_{+}(z)\right)+\xi_{\rho} w_{+}(z)^{p-1}
\end{aligned}
$$


(recall that $u_{0} \leq w_{+}$and see hypothesis $\left(\mathrm{H}_{3}(\mathrm{vi})\right.$ ). It follows that

$$
\begin{aligned}
\left\langle A_{p}\left(u_{0}\right), \vartheta\right\rangle+\left\langle A\left(u_{0}\right), \vartheta\right\rangle & -\int_{\Omega} f\left(z, u_{0}\right) \vartheta d z \leq 0 \\
& \leq\left\langle A_{p}\left(w_{+}\right), \vartheta\right\rangle+\left\langle A\left(w_{+}\right), \vartheta\right\rangle-\int_{\Omega} f\left(z, w_{+}\right) \vartheta d z
\end{aligned}
$$

for all $\vartheta \in W^{1, p}(\Omega)$ with $\vartheta \geq 0$ (see hypothesis $\left(\mathrm{H}_{3}\right)(\mathrm{v})$ ).

Let $a(y)=|y|^{p-2} y+y$ for all $y \in \mathbb{R}^{N}$. Then $a \in C^{1}\left(\mathbb{R}^{N}\right)$ (recall $p>2$ ) and

$$
\begin{aligned}
\nabla a(y)=|y|^{p-2}(I+ & \left.(p-2) \frac{y \otimes y}{|y|^{2}}\right)+I \quad \text { for all } y \in \mathbb{R}^{N} \\
& \Rightarrow(\nabla a(y) \xi, \xi)_{\mathbb{R}^{N}} \geq\left(1+|y|^{p-2}\right)|\xi|^{2} \quad \text { for all } y, \xi \in \mathbb{R}^{N} .
\end{aligned}
$$

So, by virtue of (4.10), we can apply Theorem 2.5.2 of Pucci and Serrin [23, p. 35] (the tangency principle) and infer that

$$
u_{0}(z)<w_{+}(z) \text { for all } z \in \bar{\Omega} \quad \Rightarrow \quad u_{0} \in \operatorname{int}_{C_{0}^{1}(\bar{\Omega})}\left[0, w_{+}\right]
$$

But from (4.6) and (4.8) it is clear that $\left.\widehat{\psi}_{+}\right|_{\left[0, w_{+}\right]}=\left.\psi_{+}\right|_{\left[0, w_{+}\right]}$. So, (4.11) implies that $u_{0} \in \operatorname{int} C_{+}$is a local $C_{0}^{1}(\bar{\Omega})$-minimizer of $\psi_{+}$, hence it is also a local $W_{0}^{1, p}(\Omega)$-minimizer of $\psi_{+}$(see Proposition 2.3). This proves Claim.

By virtue of Claim, we can find $\rho \in(0,1)$ small such that

$$
\psi_{+}\left(u_{0}\right)<\inf \left[\psi_{+}(u):\left\|u-u_{0}\right\|=\rho\right]=m_{+}
$$

(see Aizicovici, Papageorgiou and Staicu [1], proof of Proposition 29).

Hypothesis $\left(\mathrm{H}_{3}\right)$ (ii) implies that for any $\widetilde{u} \in \operatorname{int} C_{+}$, we have

$$
\psi_{+}(t \widetilde{u}) \rightarrow-\infty \quad \text { as } t \rightarrow+\infty .
$$

Then (4.7), (4.12) and (4.13) permit the use of Theorem 2.1 (the mountain pass theorem) and obtain $\widehat{u} \in W_{0}^{1, p}(\Omega)$ such that

$$
\widehat{u} \in K_{\psi_{+}} \quad \text { and } \quad m_{+} \leq \psi_{+}(\widehat{u}) \text {. }
$$

From (4.12) and (4.14), we see that $\widehat{u} \neq u_{0}$. Also, since $\widehat{u} \in K_{\psi_{+}}$(see (4.14)), we have that

$$
A_{p}(\widehat{u})+A(\widehat{u})=N_{g_{+}}(\widehat{u}) .
$$

Acting on (4.15) with $\left(u_{0}-\widehat{u}\right)^{+} \in W_{0}^{1, p}(\Omega)$ and using (4.6), as before we obtain that $u_{0} \leq \widehat{u}$ and so $\widehat{u}$ is a positive solution of problem (1.1) (see (4.6) and (4.15)). As before the nonlinear regularity theory implies that $\widehat{u} \in \operatorname{int} C_{+}$. 
Let $\rho=\|\widehat{u}\|_{\infty}$ and let $\xi_{\rho}>0$ be as postulated by hypothesis $\left(\mathrm{H}_{3}\right)(\mathrm{vi})$. Then for $\widehat{\xi}_{\rho}>\xi_{\rho}$ we have

$$
\begin{aligned}
& -\Delta_{p} u_{0}(z)-\Delta u_{0}(z)+\widehat{\xi}_{\rho} u_{0}(z)^{p-1}=f\left(z, u_{0}(z)\right)+\widehat{\xi}_{\rho} u_{0}(z)^{p-1} \\
& \leq f(z, \widehat{u}(z))+\widehat{\xi}_{\rho} \widehat{u}(z)^{p-1} \quad\left(\text { since } u_{0} \leq \widehat{u}, \text { see hypothesis }\left(\mathrm{H}_{3}\right)(\mathrm{vi})\right) \\
& =-\Delta_{p} \widehat{u}(z)-\Delta \widehat{u}(z)+\widehat{\xi}_{\rho} \widehat{u}(z)^{p-1} \quad \text { a.e. in } \Omega .
\end{aligned}
$$

Once again the tangency principle of Pucci and Serrin [23, Theorem 2.5.2, p. 35] implies that $u_{0}(z)<\widehat{u}(z)$ for all $z \in \Omega$. Hence, from (4.16) and Proposition 2.4 (recall $\widehat{\xi}_{\rho}>\xi_{\rho}$ ) we infer that $\widehat{u}-u_{0} \in \operatorname{int} C_{+}$.

Similarly, for the second negative solution we introduce the truncation

$$
g_{-}(z, x)= \begin{cases}f(z, x) & \text { if } x \leq v_{0}(z) \\ f\left(z, v_{0}(z)\right) & \text { if } v_{0}(z)<x\end{cases}
$$

This is a Carathéodory function. We set

$$
G_{-}(z, x)=\int_{0}^{x} g_{-}(z, s) d s
$$

and introduce the $C^{1}$-functional $\psi_{-}: W_{0}^{1, p}(\Omega) \rightarrow \mathbb{R}$ defined by

$$
\psi_{-}(u)=\frac{1}{p}\|D u\|_{p}^{p}+\frac{1}{2}\|D u\|_{2}^{2}-\int_{\Omega} G_{-}(z, u(z)) d z \quad \text { for all } u \in W_{0}^{1, p}(\Omega) .
$$

Working as above, this time using the functional $\psi_{-}$, we produce a second negative solution $\widehat{v} \in-\operatorname{int} C_{+} \operatorname{such}$ that $v_{0}-\widehat{v} \in \operatorname{int} C_{+}$.

Next we look for nodal (sign changing) solutions. We will employ tools from the Morse theory.

Let $d_{m}=\operatorname{dim} \bigoplus_{i=1}^{m} E\left(\widehat{\lambda}_{i}(2)\right)$ (recall that $E\left(\widehat{\lambda}_{i}(2)\right)$ denotes the eigenspace of $\left(-\Delta, H_{0}^{1}(\Omega)\right)$ corresponding to the eigenvalue $\widehat{\lambda}_{i}(2)$; see Section 2$)$.

Proposition 4.5. If hypotheses $\left(\mathrm{H}_{3}\right)$ hold, then $C_{k}(\varphi, 0)=\delta_{k, d_{m}} \mathbb{Z}$ for all $k \geq 0$.

Proof. Let $\tau: W_{0}^{1, p}(\Omega) \rightarrow \mathbb{R}$ be the $C^{2}$-functional defined by

$$
\tau(u)=\frac{1}{p}\|D u\|_{p}^{p}+\frac{1}{2}\|D u\|_{2}^{2}-\frac{1}{2} \int_{\Omega} f_{x}^{\prime}(z, 0) u(z)^{2} d z \quad \text { for all } u \in W_{0}^{1, p}(\Omega) .
$$

We consider the affine homotopy

$$
h(t, u)=(1-t) \varphi(u)+t \tau(u) \quad \text { for all }(t, u) \in[0,1] \times W_{0}^{1, p}(\Omega) .
$$

Suppose that we can find $\left\{t_{n}\right\}_{n \geq 1} \subseteq[0,1]$ and $\left\{u_{n}\right\}_{n \geq 1} \subseteq W_{0}^{1, p}(\Omega)$ such that (4.17) $t_{n} \rightarrow t \in[0,1], u_{n} \rightarrow 0$ in $W_{0}^{1, p}(\Omega)$ and $h_{u}^{\prime}\left(t_{n}, u_{n}\right)=0$ for all $n \geq 1$.

We have

$$
A_{p}\left(u_{n}\right)+A\left(u_{n}\right)=\left(1-t_{n}\right) N_{f}\left(u_{n}\right)+t_{n} f_{x}^{\prime}(\cdot, 0) u_{n} \quad \text { for all } n \geq 1
$$


Let $y_{n}=u_{n} /\left\|u_{n}\right\|, n \geq 1$. Then $\left\|y_{n}\right\|=1$ for all $n \geq 1$ and so we may assume that

$$
y_{n} \rightarrow y \quad \text { in } W_{0}^{1, p}(\Omega) \quad \text { and } \quad y_{n} \rightarrow y \quad \text { in } L^{r}(\Omega) .
$$

From (4.18) we have

$$
\left\|u_{n}\right\|^{p-2} A_{p}\left(y_{n}\right)+A\left(y_{n}\right)=\left(1-t_{n}\right) \frac{N_{f}\left(u_{n}\right)}{\left\|u_{n}\right\|}+t_{n} f_{x}^{\prime}(\cdot, 0) y_{n}
$$

for all $n \geq 1$. From hypotheses $\left(\mathrm{H}_{3}\right)$ (i),(iv), we have

$$
|f(z, x)| \leq c_{10}\left(|x|+|x|^{r-1}\right) \quad \text { for a.a. } z \in \Omega \text {, all } x \in \mathbb{R} \text {, some } c_{10}>0 .
$$

Note that (4.18) and Ladyzhenskaya and Uraltseva [18, p. 286], imply that $\left\{u_{n}\right\}_{n \geq 1} \subseteq L^{\infty}(\Omega)$ is bounded. So, it follows that $\left\{N_{f}\left(u_{n}\right) /\left\|u_{n}\right\|\right\}_{n \geq 1} \subseteq L^{r}(\Omega)$ is bounded. Thus by passing to a subsequence if necessary and using hypothesis $\left(\mathrm{H}_{3}\right)(\mathrm{iv})$, we have

$$
\frac{N_{f}\left(u_{n}\right)}{\left\|u_{n}\right\|} \stackrel{w}{\longrightarrow} f_{x}^{\prime}(\cdot, 0) y \quad \text { in } L^{r}(\Omega) \text { as } n \rightarrow \infty .
$$

Since $\left\{A_{p}\left(y_{n}\right)\right\}_{n \geq 1} \subseteq W^{-1, p^{\prime}}(\Omega)$ is bounded (see Proposition 2.2) and $\left\|u_{n}\right\|^{p-2} \rightarrow 0$ (recall $p>2$ and see (4.17)), we have

$$
\left\|u_{n}\right\|^{p-2} A_{p}\left(y_{n}\right) \rightarrow 0 \text { in } W^{-1, p^{\prime}}(\Omega) \text { as } n \rightarrow \infty .
$$

So, if in (4.20) we pass to the limit as $n \rightarrow \infty$ and use (4.21) and (4.22), then

$$
A(y)=f_{x}^{\prime}(\cdot, 0) y \quad \Rightarrow \quad-\Delta y(z)=f_{x}^{\prime}(z, 0) y(z) \text { a.e. in } \Omega,\left.y\right|_{\partial \Omega}=0 .
$$

Hypothesis $\left(\mathrm{H}_{3}\right)$ (iv) implies that $y=0$. On the other hand, from (4.20) and Theorem 2.1 of Lieberman [19], we know that there exist $\alpha \in(0,1)$ and $M>0$ such that

$$
y_{n} \in C_{0}^{1, \alpha}(\bar{\Omega}) \quad \text { and } \quad\left\|y_{n}\right\|_{C_{0}^{1, \alpha}(\bar{\Omega})} \leq M \quad \text { for all } n \geq 1 .
$$

Exploiting the compact embedding of $C_{0}^{1, \alpha}(\bar{\Omega})$ into $C_{0}^{1}(\bar{\Omega})$ and using (4.19), we have

$$
y_{n} \rightarrow y \text { in } C_{0}^{1}(\bar{\Omega}) \quad \Rightarrow \quad\|y\|=1,
$$

contradiction since $y=0$. So, (4.17) cannot occur and then the homotopy invariance of the critical groups implies that

$$
C_{k}(\varphi, 0)=C_{k}(\tau, 0) \text { for all } k \geq 0 .
$$

From Theorem 1 of Cingolani and Vannella [8], we have

$$
C_{k}(\tau, 0)=\delta_{k, d_{m}} \mathbb{Z} \text { for all } k \geq 0 \quad \Rightarrow \quad C_{k}(\varphi, 0)=\delta_{k, d_{m}} \mathbb{Z} \text { for all } k \geq 0
$$
(see (4.24)).

Proposition 4.6. Assume that hypotheses $\left(\mathrm{H}_{3}\right)(\mathrm{i})-(\mathrm{iii})$ hold and $\inf \varphi\left(K_{\varphi}\right)$ $>-\infty$. Then $C_{k}(\varphi, \infty)=0$ for all $k \geq 0$. 
Proof. Note that $\varphi$ satisfies the C-condition (see Aizicovici, Papageorgiou and Staicu [2]). Hypotheses $\left(\mathrm{H}_{3}\right)$ (i), (ii) imply that given $\xi>0$, we can find $c_{11}=c_{11}(\xi)>0$ such that

$$
F(z, x) \geq \frac{\xi}{p}|x|^{p}-c_{11} \quad \text { for a.a. } z \in \Omega, \text { all } x \in \mathbb{R} .
$$

Let $u \in \partial B_{1}=\left\{u \in W_{0}^{1, p}(\Omega):\|u\|=1\right\}$ and $t>0$. Then

$$
\varphi(t u) \leq \frac{t^{p}}{p}\left[1-\xi\|u\|_{p}^{p}\right]+\frac{t^{2}}{2}\|D u\|_{2}^{2}+c_{11}|\Omega|_{N} .
$$

Choosing $\xi>1 /\|u\|_{p}^{p}$ and since $p>2$, we infer that

$$
\varphi(t u) \rightarrow-\infty \text { as } t \rightarrow+\infty \text {. }
$$

By virtue of hypotheses $\left(\mathrm{H}_{3}\right)(\mathrm{i})$,(iii), we can find $\eta_{1} \in\left(0, \eta_{0}\right)$ and $c_{12}>0$ such that

$$
f(z, x) x-p F(z, x) \geq \eta_{1}|x|^{\tau}-c_{12} \text { for a.a. } z \in \Omega \text {, all } x \in \mathbb{R} .
$$

Then for all $v \in W_{0}^{1, p}(\Omega)$, we have (see (4.27))

$$
\int_{\Omega}[p F(z, v)-f(z, v) v] d z \leq-\eta_{1}\|v\|_{\tau}^{\tau}+c_{12}|\Omega|_{N} .
$$

Let $\vartheta<\min \left[-c_{12}|\Omega|_{N}-1, \inf \varphi\left(K_{\varphi}\right)\right]$. From (4.26), we see that for every $u \in$ $W_{0}^{1, p}(\Omega) \backslash\{0\}$ we can find $t \geq 1$ such that

$$
\varphi(t u) \leq \frac{\vartheta}{p} .
$$

Then, for such big $t \geq 1$, we have

$$
\begin{aligned}
& \frac{d}{d t} \varphi(t u)=\left\langle\varphi^{\prime}(t u), u\right\rangle \\
& =\frac{1}{t}\left[t^{p}\|D u\|_{p}^{p}+t^{2}\|D u\|_{2}^{2}-\int_{\Omega} f(z, t u)(t u) d z\right] \\
& \leq \frac{1}{t}\left[t^{p}\|D u\|_{p}^{p}+t^{2}\|D u\|_{2}^{2}-\int_{\Omega} p F(z, t u) d z+c_{12}|\Omega|_{N}\right] \\
& \text { (see }(4.28)) \\
& \leq \frac{1}{t}\left[p \varphi(t u)+c_{12}|\Omega|_{N}\right] \\
& \text { (since } p>2 \text { ) } \\
& \leq \frac{1}{t}\left[\vartheta+c_{12}|\Omega|_{N}\right]<0 .
\end{aligned}
$$

Given $u \in W_{0}^{1, p}(\Omega) \backslash\{0\}$, let $\tau(u) \geq 1$ be the first $t \geq 1$ such that (4.29) holds. Then by virtue of (4.30) and the implicit function theorem, we have $\tau \in C^{1}\left(W_{0}^{1, p}(\Omega) \backslash\{0\}\right)$. Let $h \in C\left([0,1] \times\left(W_{0}^{1, p}(\Omega) \backslash\{0\}\right), W_{0}^{1, p}(\Omega) \backslash\{0\}\right)$ be defined by

$$
h(t, u)=(1-t+t \tau(u)) u \quad \text { for all }(t, u) \in[0,1] \times\left(W_{0}^{1, p}(\Omega) \backslash\{0\}\right) .
$$


Note that

$$
\begin{aligned}
& h(0, u)=u, \quad h(1, u)=\tau(u) u \in \varphi^{\vartheta} \quad \text { for all } u \in W_{0}^{1, p}(\Omega) \backslash\{0\}, \\
& h(t, u)=u \quad \text { for all }(t, u) \in[0,1] \times \varphi^{\vartheta} \text {. }
\end{aligned}
$$

These facts imply that $\varphi^{\vartheta}$ is a strong deformation retract of $W_{0}^{1, p}(\Omega) \backslash\{0\}$. Using the radial retraction and Dugundji [11, Theorem 6.5, p. 325], we see that $\partial B_{1}$ is a deformation retract of $W_{0}^{1, p}(\Omega) \backslash\{0\}$. It follows that the sets $\varphi^{\vartheta}$ and $\partial B_{1}$ are homotopy equivalent and so we have

$$
H_{k}\left(W_{0}^{1, p}(\Omega), \partial B_{1}\right)=H_{k}\left(W_{0}^{1, p}(\Omega), \varphi^{\vartheta}\right) \text { for all } k \geq 0 .
$$

The choice of $\vartheta$ implies that

$$
H_{k}\left(W_{0}^{1, p}(\Omega), \varphi^{\vartheta}\right)=C_{k}(\varphi, \infty) \text { for all } k \geq 0 .
$$

Also, since $W_{0}^{1, p}(\Omega)$ is infinite dimensional, the boundary $\partial B_{1}$ is contractible in itself (see, for example, Gasinski and Papageorgiou [15, p. 691]). Therefore

$$
H_{k}\left(W_{0}^{1, p}(\Omega), \partial B_{1}\right)=0 \text { for all } k \geq 0 .
$$

From (4.31), (4.32) and (4.33) we conclude that $C_{k}(\varphi, \infty)=0$ for all $k \geq 0$.

Next we compute the critical groups of the functional $\varphi$ at $\widehat{u} \in K_{\varphi}$ and at $\widehat{v} \in K_{\varphi}$.

Proposition 4.7. If hypotheses $\left(\mathrm{H}_{3}\right)$ hold and $K_{\varphi}$ is finite, then $C_{k}(\varphi, \widehat{u})=$ $C_{k}(\varphi, \widehat{v})=\delta_{k, 1} \mathbb{Z}$ for all $k \geq 0$.

Proof. We do the proof for the pair $(\varphi, \widehat{u})$, the proof for the pair $(\varphi, \widehat{v})$ being similar. Let $\psi_{+}: W_{0}^{1, p}(\Omega) \rightarrow \mathbb{R}$ be the functional introduced in the proof of Proposition 4.4. We consider the homotopy $h_{+}:[0,1] \times W_{0}^{1, p}(\Omega) \rightarrow W_{0}^{1, p}(\Omega)$ defined by

$$
h_{+}(t, u)=(1-t) \varphi(u)+t \psi_{+}(u) \quad \text { for all }(t, u) \in[0,1] \times W_{0}^{1, p}(\Omega) .
$$

Suppose we can find $\left\{t_{n}\right\}_{n \geq 1} \subseteq[0,1]$ and $\left\{u_{n}\right\}_{n \geq 1} \subseteq W_{0}^{1, p}(\Omega)$ such that (4.34) $t_{n} \rightarrow t \in[0,1], u_{n} \rightarrow \widehat{u}$ in $W_{0}^{1, p}(\Omega)$ and $\left(h_{+}\right)_{u}^{\prime}\left(t_{n}, u_{n}\right)=0$ for all $n \geq 1$. From (4.34) we have

$$
-\Delta_{p} u_{n}(z)-\Delta u_{n}(z)=\left(1-t_{n}\right) f\left(z, u_{n}(z)\right)+t_{n} g_{+}\left(z, u_{n}(z)\right)
$$

for almost all $z \in \Omega$. From Ladyzhenskaya and Uraltseva [18, p. 286], we know that there exists $M_{8}>0$ such that $\left\|u_{n}\right\|_{\infty} \leq M_{8}$ for all $n \geq 1$. Then applying Theorem 2.1 of Lieberman [19], we can find $\alpha \in(0,1)$ and $M_{9}>0$ such that

$$
u_{n} \in C_{0}^{1, \alpha}(\bar{\Omega}) \quad \text { and } \quad\left\|u_{n}\right\|_{C_{0}^{1, \alpha}(\bar{\Omega})} \leq M_{9} \text { for all } n \geq 1 .
$$

The compact embedding of $C_{0}^{1, \alpha}(\bar{\Omega})$ into $C_{0}^{1}(\bar{\Omega})$ and (4.34) imply

$$
u_{n} \rightarrow \widehat{u} \quad \text { in } C_{0}^{1}(\bar{\Omega}) \text { as } n \rightarrow \infty \text {. }
$$


From Proposition 4.4 we know that $\widehat{u}-u_{0} \in \operatorname{int} C_{+}$. Therefore we can find $n_{0} \geq 1$ such that $u_{n} \geq u_{0}$ for all $n \geq n_{0}$. Then from (4.6) and (4.35) it follows that $\left\{u_{n}\right\}_{n \geq n_{0}} \subseteq K_{\varphi}$, which contradicts our hypothesis that $K_{\varphi}$ is finite. Therefore, we can find $\rho \in(0,1)$ small such that $\widehat{u} \in \operatorname{int} C_{+}$is the only critical point of $\left\{h_{+}(t, \cdot)\right\}_{t \in[0,1]}$ in $\bar{B}_{\rho}(\widehat{u})=\left\{u \in W_{0}^{1, p}(\Omega):\|u-\widehat{u}\| \leq \rho\right\}$. Then the homotopy invariance of critical groups implies that

$$
C_{k}(\varphi, \widehat{u})=C_{k}\left(\psi_{+}, \widehat{u}\right) \quad \text { for all } k \geq 0 .
$$

From the proof of Proposition 4.4, we know that $\widehat{u} \in \operatorname{int} C_{+}$is a critical point of mountain pass type for the functional $\psi_{+}$. Therefore

$$
C_{1}\left(\psi_{+}, \widehat{u}\right) \neq 0 \quad \Rightarrow \quad C_{1}(\varphi, \widehat{u}) \neq 0
$$

(see (4.36)). Since $\varphi \in C^{2}\left(W_{0}^{1, p}(\Omega)\right.$ ) (see hypotheses $\left(\mathrm{H}_{3}\right)$ ), from (4.37) and Papageorgiou and Smyrlis [22] (see also Papageorgiou and Rădulescu [21]), we have

$$
C_{k}(\varphi, \widehat{u})=\delta_{k, 1} \mathbb{Z} \quad \text { for all } k \geq 0 .
$$

In a similar fashion, we show that $C_{k}(\varphi, \widehat{v})=\delta_{k, 1} \mathbb{Z}$ for all $k \geq 0$.

Our strategy in order to generate nodal solutions is the following. First we show that problem (1.1) admits extremal constant sign solutions, that is there exist a smallest positive solution $u_{+} \in \operatorname{int} C_{+}$and a biggest negative solution $v_{-} \in-\operatorname{int} C_{+}$. Having the extremal constant sign solutions, we focus on the order interval $\left[v_{-}, u_{+}\right]=\left\{u \in W_{0}^{1, p}(\Omega): v_{-}(z) \leq u(z) \leq u_{+}(z)\right.$ almost everywhere in $\left.\Omega\right\}$. Through a combination of variational methods and of Morse theory, we show that the problem has a nontrivial solution in $\left[v_{-}, u_{+}\right]$distinct from $v_{-}$and $u_{+}$. The extremality of $v_{-}$and $u_{+}$, implies that this solution must be nodal. The nonhomogeneity of the differential operator, is the source of difficulties in the execution of this solution strategy. In this respect, the unilateral growth estimate in (4.1), will help us to overcome these difficulties. So, motivated by (4.1), we consider the following auxiliary Dirichlet problem:

$$
-\Delta_{p} u(z)-\Delta u(z)=\xi_{0} u(z)-\xi_{1}|u(z)|^{r-2} u(z) \quad \text { in } \Omega,\left.u\right|_{\partial \Omega}=0 .
$$

Proposition 4.8. Problem (4.38) admits a unique positive solution $u_{*} \in$ $\operatorname{int} C_{+}$and since the equation in (4.38) is odd, $v_{*}=-u_{*} \in-\operatorname{int} C_{+}$is the unique negative solution of (4.38).

Proof. Let $\mu_{+}: W_{0}^{1, p}(\Omega) \rightarrow \mathbb{R}$ be the $C^{1}$-functional defined by

$$
\mu_{+}(u)=\frac{1}{p}\|D u\|_{p}^{p}+\frac{1}{2}\|D u\|_{2}^{2}-\frac{\xi_{0}}{2}\left\|u^{+}\right\|_{2}^{2}+\frac{\xi_{1}}{p}\left\|u^{+}\right\|_{r}^{r} \quad \text { for all } u \in W_{0}^{1, p}(\Omega) .
$$

Since $r>p>2$, it is clear that $\mu_{+}$is coercive. Also, it is sequentially weakly lower semicontinuous. So, we can find $u_{*} \in W_{0}^{1, p}(\Omega)$ such that

$$
\mu_{+}\left(u_{*}\right)=\inf \left[\mu_{+}(u): u \in W_{0}^{1, p}(\Omega)\right] .
$$


Since $\xi_{0}>\widehat{\lambda}_{1}(2)$ and $p>2$, we see that for $t \in(0,1)$ small enough, we have

$$
\mu_{+}\left(t \widehat{u}_{1}(2)\right)<0 \quad \Rightarrow \quad \mu_{+}\left(u_{*}\right)<0=\mu_{+}(0)
$$

(see (4.39)), hence $u_{*} \neq 0$. From (4.39) we have

$$
\mu_{+}^{\prime}\left(u_{*}\right)=0 \quad \Rightarrow \quad A_{p}\left(u_{*}\right)+A\left(u_{*}\right)=\xi_{0} u_{*}^{+}-\xi_{1}\left(u_{*}^{+}\right)^{r-1} .
$$

On (4.40) we act with $-u_{*}^{-} \in W_{0}^{1, p}(\Omega)$ and obtain $u_{*} \geq 0, u_{*} \neq 0$. So, it follows that $u_{*}$ is a positive solution of the auxiliary problem (4.38). The nonlinear regularity theory (see [19]) and the nonlinear maximum principle (see Pucci and Serrin $\left[23\right.$, pp. 111, 120]) imply that $u_{*} \in \operatorname{int} C_{+}$.

We need to show the uniqueness of this positive solution. To this end, let $G_{0}(t)=t^{p} / p+t^{2} / 2$ for all $t \geq 0$. We set $G(y)=G_{0}(|y|)$ and consider the integral functional $j: L^{1}(\Omega) \rightarrow \overline{\mathbb{R}}=\mathbb{R} \cup\{+\infty\}$ defined by

$$
j(u)= \begin{cases}\int_{\Omega} G\left(D u^{1 / 2}\right) d z & \text { if } u \geq 0, u^{1 / 2} \in W_{0}^{1, p}(\Omega), \\ +\infty & \text { otherwise. }\end{cases}
$$

We claim that $j(\cdot)$ is convex. So, let $u_{1}, u_{2} \in \operatorname{dom} j=\left\{u \in W_{0}^{1, p}(\Omega): j(u)<\right.$ $+\infty\}$ and let $v_{1}=u_{1}^{1 / 2}, v_{2}=u_{2}^{1 / 2} \in W_{0}^{1, p}(\Omega)$. We define

$$
v_{3}=\left((1-t) u_{1}+t u_{2}\right)^{1 / 2} \quad \text { with } t \in[0,1] .
$$

From Lemma 1 of Benguria, Brezis and Lieb [6] (see also Diaz and Saa [10, Lemma 1]), we have

$$
\left|D v_{3}(z)\right| \leq\left[(1-t)\left|D v_{1}(z)\right|^{2}+t\left|D v_{2}(z)\right|^{2}\right]^{1 / 2} \quad \text { for a.a. } z \in \Omega \text {. }
$$

Note that $t \rightarrow G_{0}(t)$ is increasing and $t \rightarrow G_{0}\left(t^{1 / 2}\right)$ is convex on $[0,+\infty]$. So, we obtain

$$
\begin{aligned}
& G_{0}\left(\left|D v_{3}(z)\right|\right) \\
& \quad \leq G_{0}\left(\left[(1-t)\left|D v_{1}(z)\right|^{2}+t\left|D v_{2}(z)\right|^{2}\right]^{1 / 2}\right) \quad\left(\text { the monotonicity of } G_{0}(\cdot)\right) \\
& \leq(1-t) G_{0}\left(\left|D v_{1}(z)\right|^{2}\right)+t G_{0}\left(\left|D v_{2}(z)\right|\right) \quad\left(\text { the convexity of } t \rightarrow G_{0}\left(t^{1 / 2}\right)\right) \\
& \Rightarrow G\left(D v_{3}(z)\right) \leq(1-t) G\left(D v_{1}(z)\right)+t G\left(D v_{2}(z)\right) \quad \text { a.e. in } \Omega \\
& \Rightarrow j(\cdot) \quad \text { is convex. }
\end{aligned}
$$

Let $u, v$ be two positive solutions of auxiliary problem (4.38). Then from the first part of the proof we have $u, v \in \operatorname{int} C_{+}$. So, for all $h \in C_{0}^{1}(\bar{\Omega})$ and for $t \in[0,1]$ small, we have $u^{2} \pm t h, v^{2} \pm t h \in \operatorname{dom} j$. Then by the chain rule, we have

$$
\begin{aligned}
& j^{\prime}\left(u^{2}\right)(h)=\left\langle A_{p}(u)+A(u), \frac{h}{u}\right\rangle, \\
& j^{\prime}\left(v^{2}\right)(h)=\left\langle A_{p}(v)+A(v), \frac{h}{v}\right\rangle \text { for all } h \in W_{0}^{1, p}(\Omega)
\end{aligned}
$$


(recall $C_{0}^{1}(\bar{\Omega})$ is dense in $W_{0}^{1, p}(\Omega)$ ). The convexity of $j$, implies the monotonicity of $j^{\prime}(y)(\cdot)$ for all $y \in \operatorname{dom} j$. Hence

$$
\begin{aligned}
0 & \leq\left\langle j^{\prime}\left(u^{2}\right)-j^{\prime}\left(v^{2}\right), u^{2}-v^{2}\right\rangle \\
& =\int_{\Omega}\left(\frac{-\Delta_{p} u-\Delta u}{u}-\frac{-\Delta_{p} v-\Delta v}{v}\right)\left(u^{2}-v^{2}\right) d z \\
& =\int_{\Omega} \xi_{1}\left(v^{r-2}-u^{r-2}\right)\left(u^{2}-v^{2}\right) d z \leq 0 \\
& \Rightarrow u=v \Rightarrow u_{*} \in \operatorname{int} C_{+} \text {is the unique positive solution of (4.38). }
\end{aligned}
$$

Since the equation is odd, we deduce that $v_{*}=-u_{*} \in-\operatorname{int} C_{+}$is the unique negative solution of problem (4.38).

Now let $S_{+}$(resp. $S_{-}$) denote the set of positive (resp. negative) solutions of problem (1.1) belonging in the order interval $[0, w+]$ (resp. $\left.\left[w_{-}, 0\right]\right)$. From Proposition 4.3 we know that

$$
S_{+}, S_{-} \neq \emptyset \quad \text { and } \quad S_{+} \subseteq \operatorname{int} C_{+}, \quad S_{-} \subseteq-\operatorname{int} C_{+} .
$$

Proposition 4.9. If hypotheses $\left(\mathrm{H}_{3}\right)$ hold and $u \in S_{+}$(resp. $\left.v \in-S_{-}\right)$, then $u_{*} \leq u$ and $v \leq v_{*}$.

Proof. We do the proof for $u \in S_{+}$, the proof for $v \in S_{-}$being similar. We introduce the following Carathéodory function:

$$
\gamma_{+}(z, x)= \begin{cases}0 & \text { if } x<0 \\ \xi_{0} x-\xi_{1} x^{r-1} & \text { if } 0 \leq x \leq u(z), \\ \xi_{0} u(z)-\xi_{1} u(z)^{r-1} & \text { if } u(z)<x\end{cases}
$$

Let

$$
\Gamma_{+}(z, x)=\int_{0}^{x} \gamma_{+}(z, s) d s
$$

and consider the $C^{1}$-functional $\xi_{+}: W_{0}^{1, p}(\Omega) \rightarrow \mathbb{R}$ defined by

$$
\xi_{+}(u)=\frac{1}{p}\|D u\|_{p}^{p}+\frac{1}{2}\|D u\|_{2}^{2}-\int_{\Omega} \Gamma_{+}(z, u(z)) d z \quad \text { for all } u \in W_{0}^{1, p}(\Omega) .
$$

It is clear from (4.41) that $\xi_{+}$is coercive. Also, it is sequentially weakly lower semicontinuous. So, we can find $\widetilde{u}_{*} \in W_{0}^{1, p}(\Omega)$ such that

$$
\xi_{+}\left(\widetilde{u}_{*}\right)=\inf \left[\xi_{+}(u): u \in W_{0}^{1, p}(\Omega)\right] .
$$

As before (see the proof of Proposition 4.8), for $t \in(0,1)$ small (at least such that $t \widehat{u}_{1}(2) \leq u$; recall that $u \in \operatorname{int} C_{+}$and see Lemma 3.3 of Filippakis, Kristaly and Papageorgiou [14]), we have $\xi_{+}\left(t \widehat{u}_{1}(2)\right)<0$. Therefore $\xi_{+}\left(\widetilde{u}_{*}\right)<0$ (see (4.42)), hence $\widetilde{u}_{*} \neq 0$. Also, from (4.42) we have

$$
\xi_{+}^{\prime}\left(\widetilde{u}_{*}\right)=0 \quad \Rightarrow \quad A_{p}\left(\widetilde{u}_{*}\right)+A\left(\widetilde{u}_{*}\right)=N_{\gamma_{+}}\left(\widetilde{u}_{*}\right) .
$$


On (4.43) we act with $-\widetilde{u}_{*}^{-} \in W_{0}^{1, p}(\Omega)$ and obtain $\widetilde{u}_{*} \geq 0, \widetilde{u}_{*} \neq 0$. Also, we act with $\left(\widetilde{u}_{*}-u\right)^{+} \in W_{0}^{1, p}(\Omega)$ and have

$$
\begin{aligned}
& \left\langle A_{p}\left(\widetilde{u}_{*}\right),\left(\widetilde{u}_{*}-u\right)^{+}\right\rangle+\left\langle A\left(\widetilde{u}_{*}\right),\left(\widetilde{u}_{*}-u\right)^{+}\right\rangle=\int_{\Omega} \gamma_{+}\left(z, \widetilde{u}_{*}\right)\left(\widetilde{u}_{*}-u\right)^{+} d z \\
& =\int_{\Omega}\left[\xi_{0} u-\xi_{1} u^{r-1}\right]\left(\widetilde{u}_{*}-u\right)^{+} d z \quad \text { (see (4.41)) } \\
& \leq \int_{\Omega} f(z, u)\left(\widetilde{u}_{*}-u\right)^{+} d z \quad \quad(\text { see }(4.1)) \\
& =\left\langle A_{p}(u),\left(\widetilde{u}_{*}-u\right)^{+}\right\rangle+\left\langle A(u),\left(\widetilde{u}_{*}-u\right)^{+}\right\rangle \quad\left(\text { since } u \in S_{+}\right) \text {, } \\
& \Rightarrow\left\langle A_{p}\left(\widetilde{u}_{*}\right)-A_{p}(u),\left(\widetilde{u}_{*}-u\right)^{+}\right\rangle+\left\langle A\left(\widetilde{u}_{*}\right)-A(u),\left(\widetilde{u}_{*}-u\right)^{+}\right\rangle \leq 0 \\
& \Rightarrow\left|\left\{\widetilde{u}_{*}>u\right\}\right|_{N}=0 \text {, }
\end{aligned}
$$

hence $\widetilde{u}_{*} \leq u$. So, we have proved that

$$
\begin{array}{rlrl}
\widetilde{u}_{*} \in[0, u] & =\left\{y \in W_{0}^{1, p}(\Omega): 0 \leq y(z) \leq u(z)\right. & \text { for a.a. } z \in \Omega\}, \widetilde{u}_{*} \neq 0 \\
& \Rightarrow \widetilde{u}_{*} \text { is a nontrivial positive solution of problem (4.38) } \\
& \Rightarrow \widetilde{u}_{*}=u_{*} \in \operatorname{int} C_{+} & \text {(see (4.41) and (4.43)) } \\
& \Rightarrow u_{*} \leq u . & \text { (see Proposition 4.8) }
\end{array}
$$

Similarly we show that $v \leq v_{*}$.

Now we can establish the existence of extremal constant sign solutions for problem (1.1).

Proposition 4.10. If hypotheses $\left(\mathrm{H}_{3}\right)$ hold, then problem (1.1) admits a smallest positive solution $u_{+} \in \operatorname{int} C_{+}$and a biggest negative solution $v_{-} \in$ $-\operatorname{int} C_{+}$.

Proof. As in Filippakis, Kristaly and Papageorgiou [14], we can show that the set of positive solutions of (1.1) is downward directed, that is, if $u, y$ are two positive solutions, then there exists a third positive solution of (1.1) such that $w \leq u, w \leq y$. So, in order to produce the smallest positive solution, we can restrict ourselves to the set $S_{+}$. Then from Dunford and Schwartz [12, p. 336], we know that we can find $\left\{u_{n}\right\}_{n \geq 1} \subseteq S_{+}$such that

$$
\inf S_{+}=\inf _{n \geq 1} u_{n} \text {. }
$$

We have

$$
\begin{aligned}
A_{p}\left(u_{n}\right)+A\left(u_{n}\right)=N_{f}\left(u_{n}\right), u_{n} & \in\left[0, w_{+}\right] \quad \text { for all } n \geq 1 \\
& \Rightarrow\left\{u_{n}\right\}_{n \geq 1} \subseteq W_{0}^{1, p}(\Omega) \quad \text { is bounded. }
\end{aligned}
$$


So, we may assume that

$$
u_{n} \stackrel{\mathrm{w}}{\longrightarrow} u_{+} \quad \text { in } W_{0}^{1, p}(\Omega) \text { and } \quad u_{n} \rightarrow u_{+} \quad \text { in } L^{r}(\Omega) \text { as } n \rightarrow \infty .
$$

Acting on the equation in (4.44) with $u_{n}-u_{+} \in W_{0}^{1, p}(\Omega)$, passing to the limit as $n \rightarrow \infty$ and using (4.45), we obtain

(4.46) $\lim _{n \rightarrow \infty}\left[\left\langle A_{p}\left(u_{n}\right), u_{n}-u_{+}\right\rangle+\left\langle A\left(u_{n}\right), u_{n}-u_{+}\right\rangle\right]=0 \Rightarrow u_{n} \rightarrow u_{+}$in $W_{0}^{1, p}(\Omega)$

(as before, see the proof of Proposition 3.3). So, if in (4.44) we pass to the limit as $n \rightarrow \infty$ and use (4.46), then

$$
A_{p}\left(u_{+}\right)+A\left(u_{+}\right)=N_{f}\left(u_{+}\right) .
$$

From Proposition 4.9, we have $u_{*} \leq u_{n}$ for all $n \geq 1$, hence $u_{*} \leq u_{+}$(see (4.46)) and so $u_{+} \in S_{+}$and $u_{+}=\inf S_{+}$, that is $u_{+} \in \operatorname{int} C_{+}$is the smallest positive solution of problem (1.1). Similarly we produce $v_{-} \in-\operatorname{int} C_{+}$the biggest negative solution of problem (1.1).

Now we are ready for the second multiplicity theorem. It generates six nontrivial solutions, all with sign information.

THEOREM 4.11. If hypotheses $\left(\mathrm{H}_{3}\right)$ hold, then problem (1.1) admits at least six nontrivial solutions

$$
\begin{array}{ll}
u_{0}, \widehat{u} \in \operatorname{int} C_{+}, & \widehat{u}-u_{0} \in \operatorname{int} C_{+}, \\
v_{0}, \widehat{v} \in-\operatorname{int} C_{+}, & v_{0}-\widehat{v} \in \operatorname{int} C_{+},
\end{array}
$$

and $y_{0}, \widehat{y} \in C_{0}^{1}(\bar{\Omega}) \backslash\{0\}$ nodal.

Proof. The four nontrivial constant sign solutions

$$
u_{0}, \widehat{u} \in \operatorname{int} C_{+} \quad \text { and } \quad v_{0}, \widehat{v} \in-\operatorname{int} C_{+}, \quad \widehat{u}-u_{0} \in \operatorname{int} C_{+}, \quad v_{0}-\widehat{v} \in \operatorname{int} C_{+}
$$

come from Propositions 4.3 and 4.4. So, we need to produce the two nodal solutions. By virtue of Proposition 4.10 without any loss of generality, we may assume that $u_{0} \in \operatorname{int} C_{+}$and $v_{0} \in-\operatorname{int} C_{+}$are extremal. Let $g_{*}$ be the Carathéodory function defined by

$$
g_{*}(z, x)= \begin{cases}f\left(z, v_{0}(z)\right) & \text { if } x<v_{0}(z) \\ f(z, x) & \text { if } v_{-}(z) \leq x \leq u_{0}(z) \\ f\left(z, u_{0}(z)\right) & \text { if } u_{0}(z)<x\end{cases}
$$

Let

$$
G_{*}(z, x)=\int_{0}^{x} g_{*}(z, s) d s
$$

and consider the $C^{1}$-functional $\tau_{*}: W_{0}^{1, p}(\Omega) \rightarrow \mathbb{R}$ defined by

$$
\tau_{*}(u)=\frac{1}{p}\|D u\|_{p}^{p}+\frac{1}{2}\|D u\|_{2}^{2}-\int_{\Omega} G_{*}(z, u(z)) d z \quad \text { for all } u \in W_{0}^{1, p}(\Omega) .
$$


Also, we consider the positive and negative truncations of $g_{*}(z, \cdot)$, namely the Carathéodory functions

$$
g_{*}^{ \pm}(z, x)=g_{*}\left(z, \pm x^{ \pm}\right)
$$

We set

$$
G_{*}^{ \pm}(z, x)=\int_{0}^{x} g_{*}^{ \pm}(z, s) d s
$$

and consider the $C^{1}$-functionals $\tau_{*}^{ \pm}: W_{0}^{1, p}(\Omega) \rightarrow \mathbb{R}$ defined by

$$
\tau_{*}^{ \pm}(u)=\frac{1}{p}\|D u\|_{p}^{p}+\frac{1}{2}\|D u\|_{2}^{2}-\int_{\Omega} G_{*}^{ \pm}(z, u(z)) d z \quad \text { for all } u \in W_{0}^{1, p}(\Omega) .
$$

It is easy to see that

$$
K_{\tau_{*}} \subseteq\left[v_{0}, u_{0}\right], \quad K_{\tau_{*}^{+}} \subseteq\left[0, u_{0}\right], K_{\tau_{*}^{-}} \subseteq\left[v_{0}, 0\right]
$$

(see the proof of Proposition 4.3). The extremality of $u_{0}$ and $v_{0}$, implies that

$$
K_{\tau_{*}} \subseteq\left[v_{0}, u_{0}\right], \quad K_{\tau_{*}^{+}}=\left\{0, u_{0}\right\}, \quad K_{\tau_{*}^{-}}=\left\{v_{0}, 0\right\} .
$$

Claim. $u_{0} \in \operatorname{int} C_{+}$and $v_{0} \in-\operatorname{int} C_{+}$are local minimizers of the functional $\tau_{*}$.

From (4.47) it is clear that $\tau_{*}^{+}$is coercive. Also, it is sequentially weakly lower semicontinuous. So, we can find $\widetilde{u}_{0} \in W_{0}^{1, p}(\Omega)$ such that

$$
\tau_{*}^{+}\left(\widetilde{u}_{0}\right)=\inf \left[\tau_{*}^{+}(u): u \in W_{0}^{1, p}(\Omega)\right] .
$$

As before (see the proof of Proposition 4.3), we have $\tau_{*}^{+}\left(\widetilde{u}_{0}\right)<0=\tau_{*}^{+}(0)$, hence $\widetilde{u}_{*} \neq 0$. Since $\widetilde{u}_{0} \in K_{\tau_{*}^{+}}$(see (4.49)), we have $\widetilde{u}_{0}=u_{0}$ (see (4.48)). Recall that $u_{0} \in \operatorname{int} C_{+}$. Because $\left.\tau_{*}^{+}\right|_{C_{+}}=\left.\tau_{*}\right|_{C_{+}}$, it follows that $u_{0} \in \operatorname{int} C_{+}$is a local $C_{0}^{1}(\bar{\Omega})$-minimizer of $\tau_{*}$. Invoking Proposition 2.3, we infer that $u_{0} \in \operatorname{int} C_{+}$is a local $W_{0}^{1, p}(\Omega)$-minimizer of $\tau_{*}$. Similarly, for $v_{0} \in-\operatorname{int} C_{+}$, using this time the functional $\tau_{*}^{-}$. This proves Claim.

Without any loss of generality, we may assume that $\tau_{*}\left(v_{0}\right) \leq \tau_{*}\left(u_{0}\right)$ (the analysis is similar, if the opposite inequality holds). By virtue of Claim, we can find $\rho \in(0,1)$ small enough $\left(\rho<\left\|u_{0}-v_{0}\right\|\right)$ such that

$$
\tau_{*}\left(v_{0}\right) \leq \tau_{*}\left(u_{0}\right)<\inf \left[\tau_{*}(u):\left\|u-u_{0}\right\|=\rho\right]=m_{\rho}
$$

(see [1]). Since $\tau_{*}$ is coercive (see (4.47)), it satisfies the C-condition. This fact and (4.50), permit the use of Theorem 2.1 (the mountain pass theorem). So, we can find $y_{0} \in W_{0}^{1, p}(\Omega)$ such that

$$
y_{0} \in K_{\tau_{*}} \text { and } m_{\rho} \leq \tau_{*}\left(y_{0}\right) .
$$

From (4.50) and (4.51) it follows that $y_{0} \notin\left\{v_{0}, u_{0}\right\}$. If we can show that $y_{0} \neq 0$, then we infer that $y_{0}$ is nodal. Reasoning as in the proof of Proposition 4.4, using hypothesis $\left(\mathrm{H}_{3}\right)$ (vi) and Proposition 2.4, we show that

$$
u_{0}-y_{0} \in \operatorname{int} C_{+} \quad \text { and } \quad y_{0}-v_{0} \in \operatorname{int} C_{+} .
$$


Since $y_{0}$ is a critical point of $\tau_{*}$ of mountain pass type, we have

$$
C_{1}\left(\tau_{*}, y_{0}\right) \neq 0 .
$$

Note that $\left.\tau_{*}\right|_{\left[v_{0}, u_{0}\right]}=\left.\varphi\right|_{\left[v_{0}, u_{0}\right]}$ (see (4.47)). From this fact and (4.52), via a homotopy invariance argument, as in the proof of Proposition 4.7, we obtain

$$
C_{k}\left(\tau_{*}, y_{0}\right)=C_{k}\left(\varphi, y_{0}\right) \text { for all } k \geq 0 \quad \Rightarrow \quad C_{1}\left(\varphi, y_{0}\right) \neq 0
$$

(see (4.53)). But $\varphi \in C^{2}\left(W_{0}^{1, p}(\Omega)\right)$. Hence

$$
C_{k}\left(\varphi, y_{0}\right)=\delta_{k, 1} \mathbb{Z} \quad \text { for all } k \geq 0
$$

(see [22], [21]). From Proposition 4.5, we know that

$$
C_{k}(\varphi, 0)=\delta_{k, d_{m}} \mathbb{Z} \text { for all } k \geq 0, \text { with } d_{m} \geq 2
$$

(recall $m \geq 2$ ). Comparing (4.55) and (4.56), we have $y_{0} \neq 0$, hence $y_{0}$ is nodal and $y_{0} \in C_{0}^{1}(\bar{\Omega})$ (nonlinear regularity theory). From (4.54)-(4.56), we have

$$
\begin{aligned}
C_{k}\left(\tau_{*}, y_{0}\right) & =\delta_{k, 1} \mathbb{Z} & & \text { for all } k \geq 0, \\
C_{k}\left(\tau_{*}, 0\right) & =\delta_{k, d_{m}} \mathbb{Z} & & \text { for all } k \geq 0 .
\end{aligned}
$$

By virtue of Claim, we have

$$
C_{k}\left(\tau_{*}, u_{0}\right)=C_{k}\left(\tau_{*}, v_{0}\right)=\delta_{k, 0} \mathbb{Z} \quad \text { for all } k \geq 0 .
$$

Finally, the coercivity of $\tau_{*}$ implies

$$
C_{k}\left(\tau_{*}, \infty\right)=\delta_{k, 0} \mathbb{Z} \quad \text { for all } k \geq 0 .
$$

Suppose $K_{\tau_{*}}=\left\{v_{0}, u_{0}, 0, y_{0}\right\}$. Then from (4.57)-(4.60) and the Morse relation with $t=-1$ (see (2.1)), we have

$$
2(-1)^{0}+(-1)^{d_{m}}+(-1)^{1}=(-1)^{0} \Rightarrow(-1)^{d_{m}}=0,
$$

a contradiction. So, there exists $\widehat{y} \in K_{\tau_{*}}, \widehat{y} \notin\left\{v_{0}, u_{0}, 0, y_{0}\right\}$. From (4.48) it follows that $\widehat{y}$ is a second nodal solution and by nonlinear regularity, we have $\widehat{y} \in C_{0}^{1}(\bar{\Omega})$.

It is natural to ask what happens if in $\left(\mathrm{H}_{3}\right)$ (iv), $m \geq 1$. In this case the unilateral growth estimate (4.1) is not valid. Therefore, we cannot generate extremal solutions and so Theorem 4.11 fails. Nevertheless, we still have a multiplicity theorem producing five nontrivial solutions, but we are unable to determine the sign of the fifth solution.

The new hypotheses on the reaction $f$ are the following:

$\left(\mathrm{H}_{4}\right) f: \Omega \times \mathbb{R} \rightarrow \mathbb{R}$ is a measurable function such that for almost all $z \in \Omega$, $f(z, 0)=0, f(z, \cdot) \in C^{1}(\mathbb{R})$, hypotheses $\left(\mathrm{H}_{4}\right)(\mathrm{i})-(\mathrm{v})$ are the same as the corresponding hypotheses $\left(\mathrm{H}_{3}\right)(\mathrm{i})-(\mathrm{iii}),(\mathrm{v}),(\mathrm{vi})$ and 
(iv) $f_{x}^{\prime}(z, 0)=\lim _{x \rightarrow 0} \frac{f(z, x)}{x}$ uniformly for almost all $z \in \Omega$ and for some $m \geq 1$, we have

$$
\begin{aligned}
f_{x}^{\prime}(z, 0) \in\left[\widehat{\lambda}_{m}(2), \widehat{\lambda}_{m+1}(2)\right] & \text { for a.a. } z \in \Omega \\
& \text { and } \quad f_{x}^{\prime}(\cdot, 0) \not \equiv \widehat{\lambda}_{m}(2), \quad f_{x}^{\prime}(\cdot, 0) \not \equiv \lambda_{m+1}(2) .
\end{aligned}
$$

THEOREM 4.12. If hypotheses $\left(\mathrm{H}_{4}\right)$ hold, then problem (1.1) has at least five nontrivial solutions

$$
\begin{aligned}
u_{0}, \widehat{u} & \in \operatorname{int} C_{+} \quad \text { with } \widehat{u}-u_{0} \in \operatorname{int} C_{+}, \\
v_{0}, \widehat{v} & \in-\operatorname{int} C_{+} \quad \text { with } v_{0}-\widehat{v} \in \operatorname{int} C_{+}, \\
y_{0} & \in C_{0}^{1}(\bar{\Omega}) .
\end{aligned}
$$

Proof. A careful reading of the proofs of Propositions 4.3 and 4.4, reveals that by virtue of the UCP, the results remain valid under the new set of hypotheses $\left(\mathrm{H}_{4}\right)$. Therefore we can say that problem (1.1) has at least four nontrivial solutions of constant sign

$$
\begin{array}{ll}
u_{0}, \widehat{u} \in \operatorname{int} C_{+} & \text {with } \widehat{u}-u_{0} \in \operatorname{int} C_{+}, \\
v_{0}, \widehat{v} \in-\operatorname{int} C_{+} & \text {with } v_{0}-\widehat{v} \in \operatorname{int} C_{+} .
\end{array}
$$

From the proof of Proposition 4.3, we know that $u_{0}$ is a minimizer of $\widehat{\varphi}_{+}$ (see (4.3)). From (4.11) we have $u_{0} \in \operatorname{int}_{C_{0}^{1}(\bar{\Omega})}\left[0, w_{+}\right]$. Since $\left.\widehat{\varphi}_{+}\right|_{\left[0, w_{+}\right]}=\left.\varphi\right|_{\left[0, w_{+}\right]}$ (see (4.2)), it follows that $u_{0}$ is a local $C_{0}^{1}(\bar{\Omega})$-minimizer of $\varphi$, hence by Proposition 2.3 it is also a local $W_{0}^{1, p}(\Omega)$-minimizer of $\varphi$. Similarly we show that $v_{0} \in-\operatorname{int} C_{+}$too is a local minimizer of $\varphi$. Therefore, we have

$$
C_{k}\left(\varphi, u_{0}\right)=C_{k}\left(\varphi, v_{0}\right)=\delta_{k, 0} \mathbb{Z} \quad \text { for all } k \geq 0 .
$$

From the proof of Proposition 4.4, we know that $\widehat{u} \in \operatorname{int} C_{+}$is a critical point of $\psi_{+}$of mountain pass type. Hence

$$
C_{1}\left(\psi_{+}, \widehat{u}\right) \neq 0 .
$$

Since $\widehat{u}-u_{0} \in \operatorname{int} C_{+}$, via a homotopy invariance argument as in the proof of Proposition 4.7 , we show that

$$
\begin{aligned}
C_{k}\left(\psi_{+}, \widehat{u}\right) & =C_{k}(\varphi, \widehat{u}) & & \text { for all } k \geq 0 \\
\Rightarrow C_{1}(\varphi, \widehat{u}) & \neq 0 & & (\text { see }(4.62)) \\
\Rightarrow C_{k}(\varphi, \widehat{u}) & =\delta_{k, 1} \mathbb{Z} & & \text { for all } k \geq 0 \text { (see [22], [21]). }
\end{aligned}
$$

Similarly we show that

$$
C_{k}(\varphi, \widehat{v})=\delta_{k, 1} \mathbb{Z} \quad \text { for all } k \geq 0 .
$$


From Propositions 4.5 and 4.6, we have

$$
\begin{aligned}
C_{k}(\varphi, 0) & =\delta_{k, d_{m}} \mathbb{Z} & & \text { for all } k \geq 0, \\
C_{k}(\varphi, \infty) & =0 & & \text { for all } k \geq 0 .
\end{aligned}
$$

Suppose $K_{\varphi}=\left\{v_{0}, u_{0}, \widehat{v}, \widehat{u}, 0\right\}$. Then from (4.61), (4.63)-(4.66) and the Morse relation with $t=-1$ (see $(2.1))$, we have

$$
2(-1)^{0}+2(-1)^{1}+(-1)^{d_{m}}=0 \quad \Rightarrow \quad(-1)^{d_{m}}=0,
$$

a contradiction. So, there exists $y_{0} \in K_{\varphi}, y_{0} \notin\left\{v_{0}, u_{0}, \widehat{v}, \widehat{u}, 0\right\}$, hence $y_{0}$ is a fifth nontrivial solution for problem (1.1) and $y_{0} \in C_{0}^{1}(\bar{\Omega})$ (nonlinear regularity theory).

\section{REFERENCES}

[1] S. Aizicovici, N.S. Papageorgiou and V. Staicu, Degree Theory for Operators of Monotone Type and Nonlinear Elliptic Equations with Inequality Constraints, Memoirs Amer. Math. Soc. Vol 196, No. 915 (2008).

[2] _ On p-superlinear equations with a nonhomogeneous differential operator, Nonlinear Differential Equations Appl. (NoDEA) 20 (2013), 151-175.

[3] A. Ambrosetti and P. Rabinowitz, Dual variational methods in critical point theory and applications, J. Functional Anal. 14 (1973), 349-381.

[4] D. Arcoya And D. Ruiz, The Ambrosetti-Prodi problem for the p-Laplace operator, Comm. Partial Differential Equations 31 (2006), 849-865.

[5] T. Bartsch, Z. Liu And T. Weth, Nodal solutions of a p-Laplacian equation, Proc. London Math. Soc. 91 (2005), 129-152.

[6] R. Benguria, H. Brezis and E.H. Lieb, The Thomas-Fermi-von Weizsäcker theory of atoms and molecules, Comm. Math. Physics 79 (1981), 167-180.

[7] S. Cingolani and M. Degiovanni, Nontrivial solutions for $p$-Laplace equations with right hand side having p-linear growth, Comm. Partial Differential Equations 30 (2005), 1191-1203.

[8] S. Cingolani and G. Vannella, Critical groups computations on a class of Sobolev Banach spaces via Morse index, Ann. Inst. H. Poincaré Anal. Non Linéaire 20 (2003), 271-292.

[9] M. Degiovanni and S. Lancelotti, Linking over cones and nontrivial solutions for pLaplace equations with p-superlinear nonlinearity, Ann. Inst. H. Poincaré Analyse Non Linéaire 24 (2007), 907-919.

[10] J.I. Diaz AND J.E. SAA, Existence et unicité de solutions positives pour certaines équations elliptiques quasilinéaires, C.R. Acad. Sci. Paris Sér. 305 (1987), 521-524.

[11] J. DugundjI, Topology, Allyn and Bacon Inc, Boston (1966).

[12] N. Dunford and J. Schwartz, Linear Operators I. General Theory, Wiley-Interscience, New York (1958).

[13] E. Fadell and P. Rabinowitz, Generalized cohomological index theories for Lie group actions with an application to bifurcation questions in Hamiltonian systems, Invent. Math. 45 (1978), 139-174.

[14] M. Filippakis, A. Kristaly and N.S. Papageorgiou, Existence of five nonzero solutions with constant sign for a p-Laplacian equation, Discrete Cont. Dyn. Systems 24 (2009), 405-440. 
[15] L. Gasinski and N.S. Papageorgiou, Nonlinear Analysis, Chapman, Hall/CRC, Boca Raton, Fl. (2006).

[16] _ Multiple solutions for nonlinear coercive problems with a nonhomogeneous differential operator and a nonsmooth potential, Set. Valued Var. Anal. 20 (2012), 417-443.

[17] L. Iturriaga, E. Massa, J. Sachez And P. Ubilla, Positive solutions of the p-Laplacian involving a superlinear nonlinearity with zeros, J. Differential Equations 248 (2010), 309327.

[18] O. Ladyzhenskaya and N. Uraltseva, Linear and Quasilinear Elliptic Equations, Academic Press, New York (1968).

[19] G. Lieberman, Boundary regularity for solutions of degenerate elliptic equations, Nonlinear Anal. 12 (1988), 1203-1219.

[20] N.S. Papageorgiou and S. Kyritsi, Handbook of Applied Analysis, Springer, New York (2009).

[21] N.S. PAPAgeorgiou AND V.D. RĂDulescu, Qualitative phenomena for some classes of quasilinear elliptic equations with multiple resonance, Appl. Math. Optim. 69 (2014), 393-430.

[22] N.S. Papageorgiou And G. Smyrlis, On nonlinear nonhomogeneous resonant Dirichlet equations, Pacific J. Math. 264 (2013), 421-453.

[23] P. Pucci And J. Serrin, The Maximum Principle, Birkhäuser, Basel (2007).

[24] M. Sun, Multiplicity of solutions for a class of quasilinear elliptic equations at resonance, J. Math. Anal. Appl. 386 (2012), 661-668.

Manuscript received March 9, 2015

accepted July 10, 2015

Nikolaos S. Papageorgiou

National Technical University

Department of Mathematics

Zografou Campus

Athens 15780, GREECE

E-mail address: npapg@math.ntua.gr

Vicenţiu D. RĂDUlescu

Department of Mathematics

Faculty of Sciences

King Abdulaziz University

P.O. Box 80203

Jeddah 21589, SAUDI ARABIA

and

Institute of Mathematics

"Simion Stoilow" of the Romanian Academy

P.O. Box 1-764

014700 Bucharest, ROMANIA

E-mail address: vicentiu.radulescu@math.cnrs.fr

TMNA : Volume $48-2016-\mathrm{N}^{\mathrm{O}} 1$ 